\title{
Schur Algebras of Classical Groups II
}

\author{
Qunhua Liu ${ }^{1}$ \\ Mathematics Institute, University of Koeln, \\ Weyertal 86-90, 50931 Koeln, Germany. \\ Email address: qliu@math.uni-koeln.de
}

\begin{abstract}
We study Schur algebras of classical groups over an algebraically closed field of characteristic not 2. We prove that Schur algebras are generalized Schur algebras (in Donkin's sense) in types $A, C$ and $D$, while are not in type $B$. Consequently Schur algebras of types $A, C$ and $D$ are integral quasi-hereditary by Donkin $[8,10]$. By using the coalgebra approach we put Schur algebras of a fixed classical group into a certain inverse system. We find that the corresponding hyperalgebra is contained in the inverse limit as a subalgebra. Moreover in types $A, C$ and $D$, the surjections in the inverse systems are compatible with the integral quasi-hereditary structure of Schur algebras. Finally we calculate some Schur algebras with small parameters and prove Schur-Weyl duality in types $B, C$ and $D$ in a special case.
\end{abstract}

Key words: Schur algebra, Classical group, Generalized Schur algebra, Inverse system, Hyperalgebra.

\section{Introduction}

Throughout the paper $K$ is an algebraically closed field of characteristic not 2, $G$ a classical group and $G^{0}$ the corresponding group of similitudes defined over $K$. Let $E$ be the standard representation of $G$ and $G^{0}$, and $E^{\otimes r}(r \geqslant 1)$ the $r$-fold tensor space on which $G$ and $G^{0}$ act diagonally. Doty [11] has defined the corresponding Schur algebra to be the image of the representation map $K G \longrightarrow \operatorname{End}_{K}\left(E^{\otimes r}\right)$, which equals that of $K G^{0} \longrightarrow \operatorname{End}_{K}\left(E^{\otimes r}\right)$. Results in this paper generalize those in [22], where the base field is the complex number field $\mathbb{C}$. We will prove results for both classical groups and their similitudes.

The main results are the following. Firstly we compare these Schur algebras to Donkin's generalized Schur algebras [8], and prove that for both $G$ and $G^{0}$, Schur algebras are generalized

\footnotetext{
${ }^{1}$ The author acknowledges support by the AsiaLink network Algebras and Representations in China and Europe, ASI/B7-301/98/679-11, and by the Leverhulme Trust through the Academic Interchange Network Algebras, Representations and Applications.
} 
Schur algebras in types $A, C$ and $D$, see Theorem 3.11, while are not in type $B$, see Remark 3.12. Consequently by Donkin $[8,10]$, Schur algebras of types $A, C$ and $D$ are integral quasihereditary, with respect to the index sets given in Proposition 3.5 and Corollary 3.6 under the usual dominance order. This extends and reproves in a uniform way results of Green [16] in type $A$ and of Donkin [9] in type $C$.

Secondly by using the coalgebra approach, we put Schur algebras of a fixed classical group into a certain inverse system to recover information of the classical group. We find that the corresponding hyperalgebra is contained in the inverse limit as a subalgebra, see Theorem 4.8. This is motivated by earlier results in type $A$ (in quantum case) by Beilinson, Lusztig and MacPherson [1]. Moreover in types $A, C$ and $D$, the maps in the inverse systems are compatible with the integral quasi-hereditary structure of Schur algebras, see Proposition 4.10.

Thirdly we calculate and compare some Schur algebras when the parameter $n$ or $r$ is small. We also prove by explicit computation the Schur-Weyl duality for (special) orthogonal and symplectic groups with Brauer algebras when the parameter $r=2$, see Theorem 5.2.

The paper is organized as follows. Section 2 recalls the coordinate rings and polynomial representations of classical groups and their similitudes. Section 3, 4 and 5 are devoted to the three parts of our main results respectively. Section 3 determines when Schur algebras of classical groups are generalized Schur algebras. Section 4 recovers the hyperalgebra from the inverse limit of Schur algebras. Section 5 calculates some examples of Schur algebras.

The results in the paper were reported at the International Asialink Conference on Algebras and Representation Theory in Beijing Normal University in 2005. They are part of my PhD thesis Schur Algebras of Classical Groups (written in Chinese, defended in May 2007).

\section{Classical groups and their similitudes}

This section recalls the coordinate rings and the polynomial representations of the classical groups and their similitudes. The main references are Green [16] and Doty [11]. All representations, modules and comodules appearing in the paper are finite dimensional.

\section{$2.1 \quad$ Notations}

For a fixed integer $n \geqslant 1$, the full matrix algebra $\mathcal{M}_{n}(K)$ is an $n^{2}$-dimensional affine algebraic variety over $K$. Following Green [16], we write $c_{i j}: \mathcal{M}_{n}(K) \longrightarrow K$ for the coordinate function which sends an $n \times n$ matrix to its $(i, j)$-th entry $(1 \leqslant i, j \leqslant n)$. The polynomial algebra $K\left[c_{i j}\right]$, in $n^{2}$ variables, is a bialgebra with comultiplication and counit defined on generators 
by

$$
\Delta\left(c_{i j}\right)=\Sigma_{k=1}^{n} c_{i k} \otimes c_{k j}, \quad \epsilon\left(c_{i j}\right)=\delta_{i j}, \quad \forall i, j=1, \ldots, n .
$$

It is naturally graded by the degree of polynomials. Each homogeneous part $K\left[c_{i j}\right]_{r}(r \geqslant 0)$ is a subcoalgebra. An ideal $I$ of $K\left[c_{i j}\right]$ is said to be homogeneous if $I=\bigoplus_{r \geqslant 0}\left(I \cap K\left[c_{i j}\right]_{r}\right)$.

We set det $=\sum_{\sigma \in \Sigma_{n}} \operatorname{sgn}(\sigma) \prod_{i=1}^{n} c_{i, \sigma(i)}$ in $K\left[c_{i j}\right]_{n}$. It sends a matrix to its determinant. For $i, j=1,2, \ldots, n$, set $c_{1}(i, j)=\sum_{k=1}^{n} c_{k i} c_{k^{\prime} j}, c_{2}(i, j)=\sum_{k=1}^{n} c_{i k} c_{j k^{\prime}}$, and $c_{1}^{\prime}(i, j)=$ $\sum_{k=1}^{n} \epsilon(k) c_{k i} c_{k^{\prime} j}, c_{2}^{\prime}(i, j)=\sum_{k=1}^{n} \epsilon(k) c_{i k} c_{j k^{\prime}}$ in $K\left[c_{i j}\right]_{2}$, where $k^{\prime}=n+1-k, \epsilon(k)=1$ if $n$ is odd, $\epsilon(k)=1$ and $\epsilon\left(k^{\prime}\right)=-1$ if $n$ is even and $k \leqslant \frac{n}{2}$.

We write $X_{m}$ for one of the following classical types: $A_{m}(m \geqslant 1), B_{m}(m \geqslant 2), C_{m}(m \geqslant$ $2)$, and $D_{m}(m \geqslant 4)$. Let $n$ be $m+1$ in type $A_{m}, 2 m+1$ in type $B_{m}$, and $2 m$ in type $C_{m}$ and $D_{m}$. Write $c_{0}$ for the polynomial $c_{1}(1, n)$ in type $B_{m}$ and $D_{m}$, and for $c_{1}^{\prime}(1, n)$ in type $C_{m}$. Our study of the polynomial $c_{0}$ is motivated by [17] and [23].

\subsection{Classical groups and their coordinate rings}

The classical groups of type $A_{m}, B_{m}, C_{m}$ and $D_{m}$ are respectively the special linear group $S L_{m+1}=\left\{M \in \mathcal{M}_{m+1}(K): \operatorname{det}(M)=1\right\}$, the special orthogonal group $S O_{2 m+1}=\{M \in$ $\left.\mathcal{M}_{2 m+1}(K): \operatorname{det}(M)=1, M^{t r} J M=J=M J M^{t r}\right\}$, the symplectic group $S P_{2 m}=\{M \in$ $\left.\mathcal{M}_{2 m}(K): M^{t r} J^{\prime} M=J^{\prime}=M J^{\prime} M^{t r}\right\}$ and the special orthogonal group $S O_{2 m}=\{M \in$ $\left.\mathcal{M}_{2 m}(K): \operatorname{det}(M)=1, M^{\operatorname{tr}} J M=J=M J M^{\operatorname{tr}}\right\}$, where $J$ is any symmetric invertible matrix and $J^{\prime}$ is any anti-symmetric invertible matrix. By the convention in the previous subsection, $n$ is the size of matrices in the classical group of type $X_{m}$.

Remark 2.1. Since $K$ is algebraically closed of characteristic not 2, the classical groups $S O_{n}$ and $S P_{n}$ are independent of the choice of the matrices $J$ and $J^{\prime}$. We take $J$ to be the matrix with 1 's on all $\left(k, k^{\prime}\right)$-th entries $(k=1,2, \ldots, n)$ and zero elsewhere (except in §5.2), and $J^{\prime}$ with $\epsilon(k)$ on $\left(k, k^{\prime}\right)$-th entries $(k=1,2, \ldots, n)$ and zero elsewhere.

For a matrix $M=\left(m_{i j}\right)$, the condition $M^{t r} J M=J=M J M^{t r}$ is equivalent to that $\delta_{j, i^{\prime}}=c_{i j}\left(M^{t r} J M\right)=c_{1}(i, j)(M)=\sum_{k=1}^{n} m_{k i} m_{k^{\prime} j}$ and $\delta_{j, i^{\prime}}=c_{i j}\left(M J M^{t r}\right)=c_{2}(i, j)(M)=$ $\sum_{k=1}^{n} m_{i k} m_{j k^{\prime}}$. The condition $M^{t r} J^{\prime} M=J^{\prime}=M J^{\prime} M^{t r}$ is equivalent to that $\delta_{j, i^{\prime}}=c_{i j}\left(M^{t r} J^{\prime} M\right)=$ $c_{1}^{\prime}(i, j)(M)=\sum_{k=1}^{n} \epsilon(k) m_{k i} m_{k^{\prime} j}$ and $\delta_{j, i^{\prime}}=c_{i j}\left(M J^{\prime} M^{t r}\right)=c_{2}^{\prime}(i, j)(M)=\sum_{k=1}^{n} \epsilon(k) m_{i k} m_{j k^{\prime}}$.

By definition the classical group $G$ is an affine algebraic subvariety of $\mathcal{M}_{n}(K)$. The coordinate ring $K[G]$ is the restriction of $K\left[c_{i j}\right]$ to $G$. Namely, it is the quotient of $K\left[c_{i j}\right]$ factoring out the ideal $I(G)$ generated by the defining relations of $G$. This is a bialgebra with 
comultiplication $\Delta$ and counit $\epsilon$ inherited from those of $K\left[c_{i j}\right]$. Unfortunately $I(G)$ is not homogenous. But the intersection $I(G)^{0}:=\bigoplus_{r \geqslant 0}\left(I(G) \cap K\left[c_{i j}\right]_{r}\right)$ is and its homogeneous part $I(G)_{r}^{0}:=I(G) \cap K\left[c_{i j}\right]_{r}$ is a coideal of $K\left[c_{i j}\right]_{r}$. Hence the quotient $K\left[c_{i j}\right]_{r} / I(G)_{r}^{0}$ is a coalgebra, denoted by $K[G]_{r}^{0}$.

In type $A_{m}$, the ideal $I\left(S L_{n}\right)$ is generated by det -1 . In type $B_{m}$ and $D_{m}$, the ideal $I\left(S O_{n}\right)$ is generated by $\{\operatorname{det}-1\} \cup\left\{c_{1}\left(i, i^{\prime}\right)-1, c_{2}\left(i, i^{\prime}\right)-1: 1 \leqslant i \leqslant n\right\} \cup\left\{c_{1}(i, j), c_{2}(i, j)\right.$ : $\left.1 \leqslant j \neq i^{\prime} \leqslant n\right\}$. In type $C_{m}$, the ideal $I\left(S P_{n}\right)$ is generated by $\left\{c_{1}^{\prime}\left(i, i^{\prime}\right)-1, c_{2}^{\prime}\left(i, i^{\prime}\right)-1: 1 \leqslant\right.$ $i \leqslant n\} \cup\left\{c_{1}^{\prime}(i, j), c_{2}^{\prime}(i, j): 1 \leqslant j \neq i^{\prime} \leqslant n\right\}$.

Remark 2.2. As functions over $G$, polynomials in $K[G]_{r_{1}}^{0}$ and $K[G]_{r_{2}}^{0}\left(r_{1} \neq r_{2}\right)$ could coincide. For example, the determinant polynomial det in $K[G]_{n}^{0}$ equals 1 in $K[G]_{0}^{0}$. In type $B_{m}$ and $D_{m}$, polynomials $c_{1}\left(i, i^{\prime}\right)$ and $c_{2}\left(i, i^{\prime}\right)$ in $K\left[S O_{n}\right]_{2}^{0}$ equal 1 . In type $C_{m}$, polynomials $c_{1}^{\prime}\left(i, i^{\prime}\right)$ and $c_{2}^{\prime}\left(i, i^{\prime}\right)$ in $K\left[S P_{n}\right]_{2}^{0}$ equal 1 .

\subsection{Groups of similitudes and their coordinate rings}

We denote by $G^{0}$ the group of similitudes of a classical group $G$. The groups of similitudes of type $A_{m}, B_{m}, C_{m}$ and $D_{m}$ are

$$
\begin{aligned}
\left(S L_{m+1}\right)^{0} & =\left\{M \in \mathcal{M}_{m+1}(K): \operatorname{det}(M) \neq 0\right\}=G L_{m+1}(K) \\
\left(S O_{2 m+1}\right)^{0} & =\left\{M \in \mathcal{M}_{2 m+1}(K): M^{t r} J M=M J M^{t r}=s J, \operatorname{det}(M)=t, t, s \in K^{\times}\right\} \\
\left(S P_{2 m}\right)^{0} & =\left\{M \in \mathcal{M}_{2 m}(K): M^{t r} J^{\prime} M=M J^{\prime} M^{t r}=s J^{\prime}, s \in K^{\times}\right\} \\
\left(S O_{2 m}\right)^{0} & =\left\{M \in \mathcal{M}_{2 m}(K): M^{t r} J M=M J M^{t r}=s J, \operatorname{det}(M)=t, t=s^{m} \in K^{\times}\right\}
\end{aligned}
$$

respectively, where $J$ and $J^{\prime}$ are defined in Remark 2.1 and $K^{\times}=K \backslash\{0\}$.

For a matrix $M=\left(m_{i j}\right)$, the condition $M^{t r} J M=M J M^{t r}=s J$ is equivalent to that $s \delta_{j, i^{\prime}}=c_{i j}\left(M^{t r} J M\right)=c_{1}(i, j)(M)=\sum_{k=1}^{n} m_{k i} m_{k^{\prime} j}$ and $s \delta_{j, i^{\prime}}=c_{i j}\left(M J M^{t r}\right)=c_{2}(i, j)(M)=$ $\sum_{k=1}^{n} m_{i k} m_{j k^{\prime}}$. The condition $M^{t r} J^{\prime} M=M J^{\prime} M^{t r}=s J^{\prime}$ is equivalent to that $s \delta_{j, i^{\prime}}=$ $c_{i j}\left(M^{t r} J^{\prime} M\right)=c_{1}^{\prime}(i, j)(M)=\sum_{k=1}^{n} \epsilon(k) m_{k i} m_{k^{\prime} j}$ and $s \delta_{j, i^{\prime}}=c_{i j}\left(M J^{\prime} M^{t r}\right)=c_{2}^{\prime}(i, j)=$ $\sum_{k=1}^{n} \epsilon(k) m_{i k} m_{j k^{\prime}}$.

Remark 2.3. As functions over $G^{0}$, it holds in type $B_{m}$ that $(\operatorname{det})^{2}=\left(\mathrm{c}_{0}\right)^{n}$ and in type $C_{m}$ and $D_{m}$ that $\operatorname{det}=\left(\mathrm{c}_{0}\right)^{m}$.

Write $K\left\langle G^{0}\right\rangle$ for the subalgebra of the coordinate ring $K\left[G^{0}\right]$ generated by $c_{i j}$ 's. It is the quotient of $K\left[c_{i j}\right]$ factoring out the ideal $I\left\langle G^{0}\right\rangle=\left\{c \in K\left[c_{i j}\right]: c(g)=0, \forall g \in G^{0}\right\}$. This is a homogeneous ideal and each homogeneous part $I\left\langle G^{0}\right\rangle_{r}=I\left(G^{0}\right) \cap K\left[c_{i j}\right]_{r}$ is a coideal of 
$K\left[c_{i j}\right]_{r}$. Hence $K\left\langle G^{0}\right\rangle=\bigoplus_{r \geqslant 0} K\left[c_{i j}\right]_{r} / I\left\langle G^{0}\right\rangle_{r}$ is a graded algebra, and each homogeneous component $K\left\langle G^{0}\right\rangle_{r}$ is a coalgebra.

In type $A_{m}$, the ideal $I\left\langle G L_{m+1}\right\rangle=0$. In type $B_{m}$, the ideal $I\left\langle S O_{2 m+1}^{0}\right\rangle$ is generated by $\left\{c_{1}\left(i, i^{\prime}\right)-c_{1}(1, n), c_{2}\left(i, i^{\prime}\right)-c_{1}(1, n): 1 \leqslant i \leqslant n\right\} \cup\left\{c_{1}(i, j), c_{2}(i, j): 1 \leqslant j \neq i^{\prime} \leqslant n\right\}$. In type $C_{m}$, the ideal $I\left\langle S P_{2 m}^{0}\right\rangle$ is generated by $\left\{c_{1}^{\prime}\left(i, i^{\prime}\right)-c_{1}^{\prime}(1, n), c_{2}^{\prime}\left(i, i^{\prime}\right)-c_{1}^{\prime}(1, n): 1 \leqslant i \leqslant\right.$ $n\} \cup\left\{c_{1}^{\prime}(i, j), \quad c_{2}^{\prime}(i, j): 1 \leqslant j \neq i^{\prime} \leqslant n\right\}$. In type $D_{m}$, the ideal $I\left\langle S O_{2 m}^{0}\right\rangle$ is generated by $\left\{\operatorname{det}-c_{1}\left(i, i^{\prime}\right)^{m}, c_{2}\left(i, i^{\prime}\right)-c_{1}(1, n): 1 \leqslant i \leqslant n\right\} \cup\left\{c_{1}(i, j), c_{2}(i, j): 1 \leqslant j \neq i^{\prime} \leqslant n\right\}$. The next lemma follows directly from the definitions of $I(G)_{r}^{0}$ and $I\left\langle G^{0}\right\rangle_{r}$.

Lemma 2.4. For each non-negative integer $r \geqslant 0$, the coideals $I(G)_{r}^{0}$ and $I\left\langle G^{0}\right\rangle_{r}$ coincide. Hence the coalgebras $K[G]_{r}^{0}$ and $K\left\langle G^{0}\right\rangle_{r}$ coincide.

\subsection{Rational representations}

Let $H$ be a group with identity $1_{H}$. Write $K^{H}$ for the $K$-space of all functions from $H$ to $K$. The group structure of $H$ gives rise to $K$-liner maps: $\Delta: K^{H} \longrightarrow K^{H \times H}$ and $\epsilon: K^{H} \longrightarrow K$ defined by $\Delta(f)(g, h)=f(g \cdot h)$ and $\epsilon(f)=f\left(1_{H}\right)$, for any $f \in K^{H}$ and $g, h \in H$. Linear representations of $H$ are identified with modules over the group algebra $K H$.

Let $V$ be a $K H$-module with basis $\left\{v_{1}, v_{2}, \ldots, v_{l}\right\}$. There exist $r_{i j} \in K^{H}(1 \leqslant i, j \leqslant l)$ such that $h \cdot v_{j}=\sum_{i=1}^{l} r_{i j}(h) v_{i}$ for all $h \in H$. Following [16], we call $r_{i j}$ 's the coefficient functions of $V$, and call the subspace of $K^{H}$ spanned by $r_{i j}$ 's the coefficient space of $V$, denoted by $\operatorname{cf}(V)$. Write $F\left(K^{H}\right)$ for the subspace of $K^{H}$ spanned by the coefficient functions of all $K H$-modules. It is a coalgeba with comultiplication $\Delta$ and counit $\epsilon$ restricted from $K^{H}$. The coefficient space $\operatorname{cf}(V)$ is a finite dimensional subcoalgebra of $F\left(K^{H}\right)$ with $\Delta\left(r_{i j}\right)=$ $\sum_{k=1}^{l} r_{i k} \otimes r_{k j}$ and $\epsilon\left(r_{i j}\right)=\delta_{i j}$. The following Lemma is standard.

Lemma 2.5. Let $C$ be a finite dimensional coalgebra over $K$. Then the linear dual $C^{*}$ is naturally an algebra, and the module category $\bmod \left(C^{*}\right)$ can be identified with the right comodule category $\operatorname{comod}(C)$.

Let $H$ and $V$ be as above. Then $V$ is a natural right $\operatorname{cf}(V)$-comodule via $\rho^{*}: V \longrightarrow$ $V \otimes \operatorname{cf}(V)$ and $\rho^{*}\left(v_{j}\right)=\sum_{i=1}^{n} v_{i} \otimes r_{i j}$. By Lemma $2.5, V$ admits a $\operatorname{cf}(V)^{*}$-module structure as follows: an element $f \in \operatorname{cf}(V)^{*}$ acts on $V$ by the composition

$$
V \stackrel{\rho^{*}}{\longrightarrow} V \otimes \operatorname{cf}(V) \stackrel{1 \otimes f}{\longrightarrow} V \otimes K \stackrel{\simeq}{\longrightarrow} V .
$$

Clearly $V$ is faithful over $\operatorname{cf}(V)^{*}$. Namely the representation map, denoted by $\tau: \operatorname{cf}(V)^{*} \longrightarrow$ $\operatorname{End}_{K}(V)$, is injective. 
Lemma 2.6. (c.f. Lemma 1.2, [4]) Let $H$ and $V$ be as above. Then $\operatorname{cf}(V)^{*}$ equals the image of the representation map $\rho: K H \longrightarrow \operatorname{End}_{K}(V)$.

Proof. Note that the representation map $\rho$ factors through $\operatorname{cf}(V)^{*}$. More precisely, there exists an algebra map $\alpha: K H \longrightarrow \operatorname{cf}(V)^{*}$ such that $\rho=\tau \circ \alpha$. In fact, for any $a \in K H$ and any $c \in \operatorname{cf}(V) \subset F\left(K^{H}\right)$, view $c$ as a linear function over $K H$ and $\alpha(a)(c)$ is defined by the action of $c$ on $a$. Now it suffices to show the surjectivity of $\alpha$. Equivalently, for any $c \in \operatorname{cf}(V)$, if $\alpha(a)(c)=0$ for all $a \in K H$, then $c=0$. But this is clear.

When $H$ is an algebraic group over $K$, the coordinate ring $K[H]$ is a subcoalgebra of $F\left(K^{H}\right)$. A representation $V$ of $H$ is said to be rational if the coefficient space $\operatorname{cf}(V)$ is contained in $K[H]$. By Chapter 1 in [16], rational representations of $H$ are identified with right $K[H]$-comodules. In fact the right $K[H]$-comodule structure is induced by the right $\operatorname{cf}(V)$-comodule structure on $V$.

For a classical group $G$ (and its similitude $G^{0}$ ), a rational representation $V$ is called polynomial if the coefficient functions are polynomials in $c_{i j}$ 's, or equivalently the coefficient space $\operatorname{cf}(V)$ is contained in $K[G]$ (and in $K\left\langle G^{0}\right\rangle$ respectively). A polynomial representation of $G^{0}$ is called homogeneous of degree $r$, if the coefficient functions are homogeneous of degree $r$, or equivalently the coefficient space $\operatorname{cf}(V)$ is contained in $K\left\langle G^{0}\right\rangle_{r}$. By definition, all rational representations of $G$ are polynomial representations. But 'homogeneous representations' of $G$ are not well-defined by Remark 2.2 .

We write $M^{X}(n)$ for the category of polynomial representations of $G^{0}$, and $M^{X}(n, r)$ the full subcategory of homogeneous ones of degree $r$. There are equivalences of categories $M^{X}(n) \cong \operatorname{comod}\left(K\left\langle G^{0}\right\rangle\right)$, and $M^{X}(n, r) \cong \operatorname{comod}\left(K\left\langle G^{0}\right\rangle_{r}\right)$.

Lemma 2.7. The category $M^{X}(n)$ is decomposed into $\bigoplus_{r \geqslant 0} M^{X}(n, r)$. That is, any polynomial representation of $G^{0}$ is a direct sum of homogeneous ones, and there are no nontrivial homomorphisms or extensions between homogeneous representations of different degrees.

Proof. Since $K\left\langle G^{0}\right\rangle=\bigoplus_{r \geqslant 0} K\left\langle G^{0}\right\rangle_{r}$ is a direct sum of subcoalgebras, by [15] (1.6c), any polynomial representation is decomposed into a direct sum of homogeneous ones. In particular, indecomposable polynomial representations are homogeneous.

Note that subrepresentations of a homogeneous representation are homogeneous of the same degree, and so are the quotients. Suppose $f: V \longrightarrow V^{\prime}$ is nonzero, where $V$ and $V^{\prime}$ are homogeneous of degree $r$ and $r^{\prime}$ respectively. Then $\operatorname{Im}(f)$ is nonzero of degree both $r$ and $r^{\prime}$, which implies $r=r^{\prime}$. 
Now let $V_{1}$ and $V_{2}$ be homogeneous representations of degree $r_{1}$ and $r_{2}$ respectively $\left(r_{1} \neq\right.$ $\left.r_{2}\right)$. Suppose we have a short exact sequence of the form

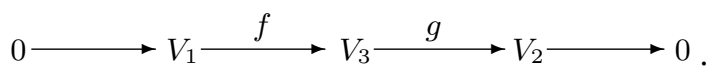

Write $V_{3}=W_{1} \oplus W_{2} \oplus W_{3}$, where $W_{1}$ and $W_{2}$ are homogeneous of degree $r_{1}$ and $r_{2}$ respectively, and $W_{3}$ contains no summand which is homogeneous of degree $r_{1}$ or $r_{2}$. Then the nonzero map $f$ maps $V_{1}$ into $W_{1}$, and $W_{1} \oplus W_{3}$ lies in the kernel of $g$. Hence the exact sequence splits and $\operatorname{Ext}^{1}\left(V_{2}, V_{1}\right)=0$.

Lemma 2.8. Let $V$ be a homogeneous polynomial representation of $G^{0}$. Then $V$ is polynomial over $G$ by restriction, and the image of the representation map $\rho_{0}: K G^{0} \longrightarrow \operatorname{End}_{K}(V)$ equals that of $\rho: K G \longrightarrow \operatorname{End}_{K}(V)$, where $\rho$ is the restriction of $\rho_{0}$ to $K G$.

Proof. The restriction to $G$ of elements in $K\left\langle G^{0}\right\rangle$ belong to $K[G]$. Hence the homogeneous representation $V$ of $G^{0}$ is polynomial over $G$. Since $K G$ is a subalgebra of $K G^{0}, \operatorname{Im}(\rho)$ is contained in $\operatorname{Im}\left(\rho_{0}\right)$ as a subalgebra.

On the other hand, given any matrix $g^{0} \in G^{0}$, write $d(\neq 0)$ for the determinant. Since $K$ is algebraically closed, there exists $\lambda \in K$ such that $\lambda^{n}=d$, where $n$ is the size of the matrices in $G^{0}$. Then $g:=\lambda^{-1} g^{0}$ belongs to $G$ since $\operatorname{det}(g)=1$. Suppose $V$ is of degree $r$. Thus $f\left(g^{0}\right)=\lambda^{r} f(g)$ for any homogeneous polynomial $f$ in $K\left[c_{i j}\right]_{r}$. It follows that $\rho_{0}\left(g^{0}\right)=$ $\rho_{0}(\lambda \cdot g)=\lambda^{r} \rho_{0}(g)=\lambda^{r} \rho(g)$ belongs to $\operatorname{Im}(\rho)$. Thus $\rho_{0}\left(G^{0}\right)$, and hence $\operatorname{Im}\left(\rho_{0}\right)=\rho_{0}\left(K G^{0}\right)$, are contained in $\operatorname{Im}(\rho)$. We have shown that $\operatorname{Im}\left(\rho_{0}\right)$ equals $\operatorname{Im}(\rho)$ as algebras.

\section{Schur algebras and generalized Schur algebras}

The main result in this section is Theorem 3.11 and Remark 3.12. Namely in contrast to type $B$, Schur algebras of type $A, C$ and $D$ are generalized Schur algebras associated to the saturated weight sets given in $§ 3.2$.

\subsection{Schur algebras of classical groups}

Recall that $X_{m}$ indicates a classical type (§2.1), $G$ the classical group of type $X_{m}, G^{0}$ the corresponding group of similitudes, and $n$ the size of matrices in $G$ and $G^{0}$. Let $E$ be the standard representation of $G$ and $G^{0}$. Namely $E$ is an $n$-dimensional column vector space over $K$ on which groups $G$ and $G^{0}$ act by matrix multiplication. For any integer $r \geqslant 1$, the groups $G$ and $G^{0}$ act diagonally on the $r$-fold tensor space $E^{\otimes r}$. 
Definition 3.1. (Doty [11]) For any integer $r \geqslant 1$, the Schur algebra of type $X_{m}$, denoted by $S^{X}(n, r)$, is defined to be the image of the representation map

$$
\rho^{r}: K G \longrightarrow \operatorname{End}_{K}\left(E^{\otimes r}\right),
$$

which equals that of

$$
\rho_{0}^{r}: K G^{0} \longrightarrow \operatorname{End}_{K}\left(E^{\otimes r}\right)
$$

For $r=0$, we define $S^{X}(n, 0)=K$.

Note that the coefficient spaces of $E^{\otimes r}$ over $G$ and $G^{0}$ are the coalgebras $K[G]_{r}^{0}$ and $K\left\langle G^{0}\right\rangle_{r}$ respectively (for definition see $\S 2.2$ and $\S 2.3$ ). Hence $E^{\otimes r}$ is a homogeneous polynomial representation of $G^{0}$ of degree $r$. By Lemma 2.8 the two images in the definition coincide.

Although we require $m \geqslant 2$ in type $B_{m}$ and $C_{m}$, and $m \geqslant 4$ in type $D_{m}$, it is actually unnecessary for the definition. For smaller positive integer $m$, the Lie group still exists as a linear subgroup of $G L_{n}$. So the corresponding Schur algebra can be defined in the same way. The case when $m=1$ will be discussed in $\S 5$.

Corollary 3.2. For any integer $r \geqslant 0$, there are algebra isomorphisms from $S^{X}(n, r)$ to the linear dual of the coalgebra $K[G]_{r}^{0}$, and to the linear dual of $K\left\langle G^{0}\right\rangle_{r}$.

It follows from Lemma 2.4 and Lemma 2.6 directly. In type $A_{m}$, the coalgebra $K\left\langle G L_{n}\right\rangle_{r}=$ $K\left[c_{i j}\right]_{r}$. Hence the Schur algebra $S^{A}(n, r)$ coincides with $S_{K}(n, r)$ defined by Green [16].

By Lemma 2.5 and Corollary 3.2, the module category $\bmod \left(S^{X}(n, r)\right)$ is equivalent to the right comodule category $\operatorname{comod}\left(K\left\langle G^{0}\right\rangle_{r}\right)$. The latter is equivalent to $M^{X}(n, r)$, the category of $r$-homogeneous polynomial representations of $G^{0}$. Indeed since $S^{X}(n, r)$ is a quotient algebra of $K G^{0}$, any $S^{X}(n, r)$-module is naturally a representation of $G^{0}$. It is in fact homogeneous of degree $r$. Conversely any such representation of $G^{0}$ arises in this way.

Corollary 3.3. There is a natural equivalence of categories between $\bmod \left(S^{X}(n, r)\right)$ and $M^{X}(n, r)$.

A representation $U$ of $G^{0}$ is said to be a sub-quotient of another representation $V$, if $U$ is isomorphic to a quotient of some submodule of $V$. By Lemma 2.7, sub-quotients of $E^{\otimes r}$ lie in $M^{X}(n, r)$ since $E^{\otimes r}$ does itself.

Proposition 3.4. Any homogeneous polynomial representation of $G^{0}$ of degree $r$ is a subquotient of $\left(E^{\otimes r}\right)^{\oplus l}$ for some $l \geqslant 1$. 
Proof. By definition 3.1, $E^{\otimes r}$ is faithful over the Schur algebra $S^{X}(n, r)$. Hence $S^{X}(n, r)$ as the regular module is a submodule of the direct sum of several copies of $E^{\otimes r}$, and so is every projective module. Take any homogeneous polynomial representation $V$ of $G^{0}$ of degree $r$. View it as an $S^{X}(n, r)$-module. Then the projective cover of $V$ is a submodule of $\left(E^{\otimes r}\right)^{\oplus l}$ for some $l \geqslant 1$. Since $V$ is a quotient of its projective cover, it is a sub-quotient of $\left(E^{\otimes r}\right)^{\oplus l}$.

\subsection{Dominant weights of Schur algebras}

Note that $E$ is a simple Weyl module. By [7], the tensor product of Weyl modules over an algebraic group has a filtration with sections being Weyl modules. Let $\pi^{X}(n, r)$ and $\pi_{0}^{X}(n, r)$ be the sets of highest weights of the Weyl modules occurred in $E^{\otimes r}$ over $G$ and $G^{0}$ respectively. They are independent of the field $K$ as Weyl modules are integrally defined. When $K=\mathbb{C}$, Weyl modules are simple and $E^{\otimes r}$ is semisimple. Hence $\pi^{X}(n, r)$ and $\pi_{0}^{X}(n, r)$ consist of highest weights of simple direct summands in $E^{\otimes r}$. By using Littelmann's path model to decompose $E^{\otimes r}$, the set $\pi^{X}(n, r)$ has been determined in [22].

Roots and dominant weights of an algebraic group can be calculated from its Lie algebra. Refer to [18] or [22] for the calculation of $G$, which can be carried over to $G^{0}$. Write $\lambda_{0}=$ $\varepsilon_{1}+\varepsilon_{2}+\ldots+\varepsilon_{n}$ and $\varepsilon_{0}=\varepsilon_{i}+\varepsilon_{i^{\prime}}$, where $i^{\prime}=n+1-i$. Thus in types $C_{m}$ and $D_{m}, n=2 m$ is even and $\varepsilon_{0}=\varepsilon_{1}+\varepsilon_{n}=\ldots=\varepsilon_{m}+\varepsilon_{m+1}, \lambda_{0}=m \varepsilon_{0}$. In type $B_{m}, n=2 m+1$ is odd and $\varepsilon_{0}=\varepsilon_{1}+\varepsilon_{n}=\ldots=\varepsilon_{m}+\varepsilon_{m+2}=2 \varepsilon_{m+1}, 2 \lambda_{0}=n \varepsilon_{0}$.

Let us denote by respectively $\Phi^{+}\left(X_{m}\right)$ and $\Lambda^{+}\left(X_{m}\right)$ the sets of positive roots and dominant weights of $G$ in type $X_{m} ; \Phi_{0}^{+}\left(X_{m}\right)$ and $\Lambda_{0}^{+}\left(X_{m}\right)$ those of $G^{0}$ in type $X_{m}$. By [22], dominant weights in $\Lambda^{+}\left(B_{m}\right)$ and $\Lambda^{+}\left(D_{m}\right)$ may have fractional coefficients, while $\pi^{X}(n, r)$ only involves integral coefficients. Hence we will only consider the set $\Lambda^{+}\left(X_{m}\right)_{\text {int }}$ of dominant weights with integral coefficients instead of $\Lambda^{+}\left(X_{m}\right)$, and similarly $\Lambda_{0}^{+}\left(X_{m}\right)_{\text {int }}$ instead of $\Lambda_{0}^{+}\left(X_{m}\right)$. When $X=A$ or $C$, we have $\Lambda^{+}\left(X_{m}\right)_{\text {int }}=\Lambda^{+}\left(X_{m}\right)$ and $\Lambda_{0}^{+}\left(X_{m}\right)_{\text {int }}=\Lambda_{0}^{+}\left(X_{m}\right)$.

We write $\mathbb{N}_{0}$ for the set of non-negative integers. In type $A_{m}(m \geqslant 1)$ :

$$
\begin{aligned}
\Phi^{+}\left(A_{m}\right) & =\Phi_{0}^{+}\left(A_{m}\right)=\left\{\varepsilon_{i}-\varepsilon_{j}: 1 \leqslant i<j \leqslant n\right\} \\
\Lambda^{+}\left(A_{m}\right) & =\left\{\sum_{i=1}^{m} a_{i} \varepsilon_{i}: a_{1} \geqslant a_{2} \geqslant \ldots \geqslant a_{m}, a_{1}, \ldots, a_{m} \in \mathbb{N}_{0}\right\} \\
\Lambda_{0}^{+}\left(A_{m}\right) & =\left\{\sum_{i=1}^{m+1} a_{i} \varepsilon_{i}: a_{1} \geqslant a_{2} \geqslant \ldots \geqslant a_{m+1}, a_{1}, \ldots, a_{m+1} \in \mathbb{Z}\right\} \\
& =\left\{\sum_{i=1}^{m} a_{i} \varepsilon_{i}+a_{0} \lambda_{0}: a_{1} \geqslant \ldots \geqslant a_{m}, a_{1}, \ldots, a_{m} \in \mathbb{N}_{0}, a_{0} \in \mathbb{Z}\right\} .
\end{aligned}
$$


In type $B_{m}(m \geqslant 2)$ :

$$
\begin{aligned}
\Phi^{+}\left(B_{m}\right) & =\left\{\varepsilon_{i} \pm \varepsilon_{j}: 1 \leqslant i<j \leqslant m ; \varepsilon_{i}: 1 \leqslant i \leqslant m\right\}, \\
\Phi_{0}^{+}\left(B_{m}\right) & =\left\{\varepsilon_{i}-\varepsilon_{j}, \varepsilon_{i}+\varepsilon_{j}-\varepsilon_{0}: 1 \leqslant i<j \leqslant m ; \varepsilon_{i}-\varepsilon_{m+1}: 1 \leqslant i \leqslant m\right\}, \\
\Lambda^{+}\left(B_{m}\right)_{\mathrm{int}} & =\left\{\sum_{i=1}^{m} a_{i} \varepsilon_{i}: a_{1} \geqslant a_{2} \geqslant \ldots \geqslant a_{m}, a_{1}, \ldots, a_{m} \in \mathbb{N}_{0}\right\}, \\
\Lambda_{0}^{+}\left(B_{m}\right)_{\mathrm{int}} & =\left\{\sum_{i=1}^{m} a_{i} \varepsilon_{i}+a_{0} \varepsilon_{m+1}: a_{1} \geqslant a_{2} \geqslant \ldots \geqslant a_{m}, a_{1}, \ldots, a_{m} \in \mathbb{N}_{0}, a_{0} \in \mathbb{Z}\right\} .
\end{aligned}
$$

In type $C_{m}(m \geqslant 2)$ :

$$
\begin{aligned}
\Phi^{+}\left(C_{m}\right) & =\left\{\varepsilon_{i} \pm \varepsilon_{j}: 1 \leqslant i<j \leqslant m ; 2 \varepsilon_{i}: 1 \leqslant i \leqslant m\right\}, \\
\Phi_{0}^{+}\left(C_{m}\right) & =\left\{\varepsilon_{i}-\varepsilon_{j}, \varepsilon_{i}+\varepsilon_{j}-\varepsilon_{0}: 1 \leqslant i<j \leqslant m ; \varepsilon_{i}-\varepsilon_{i^{\prime}}: 1 \leqslant i \leqslant m\right\}, \\
\Lambda^{+}\left(C_{m}\right) & =\left\{\sum_{i=1}^{m} a_{i} \varepsilon_{i}: a_{1} \geqslant a_{2} \geqslant \ldots \geqslant a_{m}, a_{1}, \ldots, a_{m} \in \mathbb{N}_{0}\right\}, \\
\Lambda_{0}^{+}\left(C_{m}\right) & =\left\{\sum_{i=1}^{m} a_{i} \varepsilon_{i}+a_{0} \varepsilon_{0}: a_{1} \geqslant a_{2} \geqslant \ldots \geqslant a_{m}, a_{1}, \ldots, a_{m} \in \mathbb{N}_{0}, a_{0} \in \mathbb{Z}\right\} .
\end{aligned}
$$

In type $D_{m}(m \geqslant 4)$ :

$$
\begin{aligned}
\Phi^{+}\left(D_{m}\right) & =\left\{\varepsilon_{i} \pm \varepsilon_{j}: 1 \leqslant i<j \leqslant m\right\}, \\
\Phi_{0}^{+}\left(D_{m}\right) & =\left\{\varepsilon_{i}-\varepsilon_{j}, \varepsilon_{i}+\varepsilon_{j}-\varepsilon_{0}: 1 \leqslant i<j \leqslant m\right\}, \\
\Lambda^{+}\left(D_{m}\right)_{\text {int }} & =\left\{\sum_{i=1}^{m} a_{i} \varepsilon_{i}: a_{1} \geqslant \ldots \geqslant a_{m-1} \geqslant\left|a_{m}\right|, a_{1}, \ldots, a_{m} \in \mathbb{Z}\right\}, \\
\Lambda_{0}^{+}\left(D_{m}\right)_{\text {int }} & =\left\{\sum_{i=1}^{m} a_{i} \varepsilon_{i}+a_{0} \varepsilon_{0}: a_{1} \geqslant \ldots \geqslant a_{m-1} \geqslant\left|a_{m}\right|, a_{0}, a_{1}, \ldots, a_{m} \in \mathbb{Z}\right\} .
\end{aligned}
$$

Note that the integers $a_{1}, \ldots, a_{m-1}$ in $\Lambda^{+}\left(D_{m}\right)_{\text {int }}$ and $\Lambda_{0}^{+}\left(D_{m}\right)_{\text {int }}$ are actually non-negative.

For $n, r$ and $i$ in $\mathbb{N}_{0}$, let $\Lambda^{+}(n, r)=\left\{\left(a_{1}, a_{2}, \ldots, a_{n}\right): a_{1} \geqslant a_{2} \geqslant \ldots \geqslant a_{n}, \sum_{i=1}^{n} a_{i}=\right.$ $\left.r, a_{1}, a_{2}, \ldots, a_{n} \in \mathbb{N}_{0}\right\}$ be the set of partitions. Define $\Lambda_{i}^{+}(n, r)=\left\{\left(a_{1}, a_{2}, \ldots, a_{n}\right) \in \Lambda^{+}(n, r)\right.$ : $\left.a_{1}, \ldots, a_{n-i} \neq 0\right\}$, and $\Lambda^{ \pm}(n, r)=\left\{\left(a_{1}, a_{2} \ldots, a_{n}\right):\left(a_{1}, \ldots, a_{n-1},\left|a_{n}\right|\right) \in \Lambda^{+}(n, r)\right\}$. By convention $\Lambda^{+}(n, r)=\emptyset$ if $r<0$. As in [22], a dominant weight $\sum_{i=1}^{m} a_{i} \varepsilon_{i} \in \Lambda^{+}\left(X_{m}\right)_{\text {int }}$ is identified with a partition $\left(a_{1}, a_{2}, \ldots, a_{m}\right)$.

Proposition 3.5. (Theorem 4.5, [22]) The weight sets which describe the Schur algebras of 
classical groups are:

$$
\begin{aligned}
& \pi^{A}(n, r)=\bigcup_{i \geqslant 0} \Lambda^{+}(m, r-n i) \\
& \pi^{B}(n, r)=\bigcup_{i \geqslant 0} \Lambda^{+}(m, r-2 i) \cup \bigcup_{i \geqslant 0} \Lambda_{i}^{+}(m, r-(2 i+1)), \\
& \pi^{C}(n, r)=\bigcup_{i \geqslant 0} \Lambda^{+}(m, r-2 i), \\
& \pi^{D}(n, r)=\bigcup_{i \geqslant 0} \Lambda^{ \pm}(m, r-2 i) .
\end{aligned}
$$

By sending $\lambda_{0}$ to zero (note that $\varepsilon_{0}$ is sent to zero too in types $B, C$ and $D$ ), we obtain a surjection $\Lambda_{0}^{+}\left(X_{m}\right)_{\text {int }} \longrightarrow \Lambda^{+}\left(X_{m}\right)_{\text {int }}$, and two bijection $\Phi_{0}^{+}\left(X_{m}\right) \longrightarrow \Phi^{+}\left(X_{m}\right)$ and $\pi_{0}^{X}(n, r) \longrightarrow \pi^{X}(n, r)$. Define the degree of a dominant weight $\lambda=a_{1} \varepsilon_{1}+\ldots+a_{n} \varepsilon_{n}$ to be $\operatorname{deg}(\lambda)=a_{1}+\ldots+a_{n}$. For example $\operatorname{deg}\left(\lambda_{0}\right)=n$ and $\operatorname{deg}\left(\varepsilon_{0}\right)=2$. Then $\pi_{0}^{X}(n, r)=$ $\Lambda_{0}^{+}\left(X_{m}\right)_{\text {int }} \cap\left\{\lambda+a_{0} \lambda_{0}: \lambda \in \pi^{X}(n, r), a_{0} \in \mathbb{Q}_{0}, \operatorname{deg}(\lambda)+n a_{0}=r\right\}$, where $\mathbb{Q}_{0}$ is the set of non-negative rational numbers.

Corollary 3.6. The weight sets which describe the Schur algebras of classical similitudes are:

$$
\begin{aligned}
\pi_{0}^{A}(n, r)= & \left\{\sum_{i=1}^{n} a_{i} \varepsilon_{i}: a_{1} \geqslant a_{2} \geqslant \ldots \geqslant a_{n}, a_{1}, \ldots, a_{n} \in \mathbb{N}_{0}, \sum_{i=1}^{n} a_{i}=r\right\}, \\
\pi_{0}^{B}(n, r)= & \bigcup_{i \geqslant 0}\left\{\sum_{i=1}^{m} a_{i} \varepsilon_{i}+(2 i) \varepsilon_{m+1}:\left(a_{1}, \ldots, a_{m}\right) \in \Lambda^{+}(m, r-2 i)\right\} \cup \\
& \bigcup_{i \geqslant 0}\left\{\sum_{i=1}^{m} a_{i} \varepsilon_{i}+(2 i+1) \varepsilon_{m+1}:\left(a_{1}, \ldots, a_{m}\right) \in \Lambda_{i}^{+}(m, r-(2 i+1))\right\}, \\
\pi_{0}^{C}(n, r)= & \left\{\sum_{i=1}^{m} a_{i} \varepsilon_{i}+a_{0} \varepsilon_{0}: a_{1} \geqslant a_{2} \geqslant \ldots \geqslant a_{m}, a_{0}, a_{1}, \ldots, a_{m} \in \mathbb{N}_{0}, \sum_{i=1}^{m} a_{i}+2 a_{0}=r\right\}, \\
\pi_{0}^{D}(n, r)= & \left\{\sum_{i=1}^{m} a_{i} \varepsilon_{i}+a_{0} \varepsilon_{0}: a_{1} \geqslant \ldots \geqslant a_{m-1} \geqslant\left|a_{m}\right|, \sum_{i=1}^{m} a_{i}+2 a_{0}=r,\right. \\
& \left.a_{0}, a_{1}, \ldots, a_{m-1} \in \mathbb{N}_{0}, a_{m} \in \mathbb{Z}\right\} .
\end{aligned}
$$

Under the assumption on $K$, the coalgebras $K[G]_{r}^{0}$ and $K\left\langle G^{0}\right\rangle_{r}$ are integral (See Oehms [23] for the symplectic bideterminant basis over $\mathbb{Z}$, and Doty and $\mathrm{Hu}$ [13] for the orthogonal bideterminant basis over $\left.\mathbb{Z}\left[\frac{1}{2}\right]\right)$. Hence the Schur algebra $S^{X}(n, r)$ is integral by Corollary 3.2. In particular the dimension of $S^{X}(n, r)$ is independent of the field $K$. For a dominant weight $\lambda$ of $G$ or $G^{0}$, write $d_{\lambda}$ for the dimension of the Weyl module $W(\lambda)$ with highest weight $\lambda$. By the comparison of $\pi^{X}(n, r)$ and $\pi_{0}^{X}(n, r)$, there exists for each $\lambda \in \pi^{X}(n, r)$ a unique $\lambda^{\prime} \in \pi_{0}^{X}(n, r)$ with $\lambda^{\prime}-\lambda \in \mathbb{Q}_{0} \lambda_{0}$. The restriction of the Weyl module $W\left(\lambda^{\prime}\right)$ over $G^{0}$ to $G$ is just the Weyl module $W(\lambda)$ over $G$, and hence $d_{\lambda}=d_{\lambda^{\prime}}$. By Corollary 4.4 and Proposition 5.2 in $[22]$ we have the following. 
Corollary 3.7. The dimension of the Schur algebra $S^{X}(n, r)$ is $\sum_{\lambda \in \pi^{X}(n, r)} d_{\lambda}^{2}$, which equals $\sum_{\mu \in \pi_{0}^{X}(n, r)} d_{\mu}^{2}$. In particular, when $r=2, \operatorname{dim} S^{A}(n, 2)=\left(\begin{array}{c}n^{2}+1 \\ 2\end{array}\right), \operatorname{dim} S^{B}(n, 2)=\operatorname{dim} S^{D}(n, 2)=$ $\left(\begin{array}{c}n^{2}+1 \\ 2\end{array}\right)-(n+2)(n-1)$, and $\operatorname{dim} S^{C}(n, 2)=\left(\begin{array}{c}n^{2}+1 \\ 2\end{array}\right)-(n-2)(n+1)$.

Let $\lambda$ and $\mu$ be two dominant weights of $G$ or $G^{0}$. We say $\lambda$ is dominance bigger than $\mu$, denoted by $\lambda \unrhd \mu$, if the difference $\lambda-\mu$ is a linear combination of positive roots with non-negative integer coefficients. A subset $\pi$ of $\Lambda^{+}\left(X_{m}\right)$ or $\Lambda_{0}^{+}\left(X_{m}\right)$ is said to be saturated, if $\lambda \unrhd \mu$ and $\lambda \in \pi$ imply $\mu \in \pi$. For example $\Lambda^{+}\left(X_{m}\right)_{\text {int }}$ and $\Lambda_{0}^{+}\left(X_{m}\right)_{\text {int }}$ are saturated in $\Lambda^{+}\left(X_{m}\right)$ and $\Lambda_{0}^{+}\left(X_{m}\right)$ respectively. Given dominant weights $\lambda$ and $\mu$ in $\pi^{X}(n, r)$, let $\lambda^{\prime}$ and $\mu^{\prime}$ be the corresponding dominant weights in $\pi_{0}^{X}(n, r)$. It is clear that $\lambda \unrhd \mu$ if and only if $\lambda^{\prime} \unrhd \mu^{\prime}$. The next Lemma follows from this fact and Corollary 4.7 in [22].

Lemma 3.8. The following are equivalent for any integer $r \geqslant 1$ : (1) $\pi^{X}(n, r) \subset \Lambda^{+}\left(X_{m}\right)$ is saturated; (2) $\pi_{0}^{X}(n, r) \subset \Lambda_{0}^{+}\left(X_{m}\right)$ is saturated; (3) $X=A, C$, or $D$.

Take $X_{m}=B_{2}$ and $r=3$ for example. We have that $\pi^{B}(5,3)=\{(30),(21),(11),(10)\}=$ $\left\{3 \varepsilon_{1}, 2 \varepsilon_{1}+\varepsilon_{2}, \varepsilon_{1}+\varepsilon_{2}, \varepsilon_{1}\right\}$. Notice that $3 \varepsilon_{1} \unrhd 2 \varepsilon_{1}$ because the difference $\varepsilon_{1}$ is a positive root. But $3 \varepsilon_{1}$ belongs to $\pi^{B}(5,3)$, while $2 \varepsilon_{1}$ does not. Hence $\pi^{B}(5,3)$ is not saturated in $\Lambda^{+}\left(B_{2}\right)_{\text {int }}$. Similarly $\pi_{0}^{B}(5,3)=\left\{3 \varepsilon_{1}, 2 \varepsilon_{1}+\varepsilon_{2}, \varepsilon_{1}+\varepsilon_{2}+\varepsilon_{3}, \varepsilon_{1}+2 \varepsilon_{3}\right\}$ is not saturated in $\Lambda_{0}^{+}\left(B_{2}\right)_{\text {int }}$.

\subsection{Generalized Schur algebras}

Generalized Schur algebras were introduced by Donkin [8]. Let $H$ be a reductive algebraic group over $K$, and $\Lambda^{+}(H)$ the set of dominant weights of $H$. The coordinate ring $K[H]$ has a natural left $H$-module structure given by $(g \cdot c)(h)=c(h g)$, for $g, h \in H$ and $c \in K[H]$.

Given a finite saturated subset $\pi$ of $\Lambda^{+}(H)$, a rational $K H$-module $V$ belongs to $\pi$ if every composition factor of $V$ has highest weight in $\pi$. Let $O_{\pi}(K[H])$ be the biggest $K H$ submodule of $K[H]$ which belongs to $\pi$. This is a finite dimensional coalgebra. The generalized Schur algebra associated to $\pi$ is by definition the linear dual of $O_{\pi}(K[H])$, denoted by $S(\pi)$. Recall that $d_{\lambda}$ denotes the dimension of the Weyl module of $H$ with highest weight $\lambda$. Write $\operatorname{rmod}(K H)$ for the category of rational $K H$-modules.

Proposition 3.9. (Donkin [8]) Let $\pi$ be a finite saturated subset of $\Lambda^{+}(H)$.

(1) The generalized Schur algebra $S(\pi)$ is finite dimensional with $\operatorname{dim} S(\pi)=\sum_{\lambda \in \pi} d_{\lambda}^{2}$.

(2) The module category $\bmod (S(\pi))$ is an extension-closed full subcategory of $\operatorname{rmod}(K H)$, consisting of those $H$-modules which belong to $\pi$.

The following lemma follows from (1.2e) and (1.2g) of [15]. 
Lemma 3.10. Let $H$ be as above and $V$ a rational $K H$-module. Then the coefficient space $\operatorname{cf}(V)$ is a $K H$-submodule of $K[H]$. Moreover $V$ and $\operatorname{cf}(V)$ share the same set of composition factors as $K H$-modules.

Theorem 3.11. In types $A_{m}(m \geqslant 1), C_{m}(m \geqslant 2)$ and $D_{m}(m \geqslant 4)$, for any integer $r \geqslant 1$, the Schur algebra $S^{X}(n, r)$ is isomorphic to the generalized Schur algebra of the classical group $G$ associated to $\pi^{X}(n, r)$, and to the generalized Schur algebra of $G^{0}$ associated to $\pi_{0}^{X}(n, r)$.

Proof. Fix a type $X_{m}$ as required and an integer $r \geqslant 1$. Write $\pi$ for $\pi^{X}(n, r)$ in the proof. We will show that $K[G]_{r}^{0}$ and $O_{\pi}(K[G])$ coincide as subcoalgebras of $K[G]$. Consequently their linear dual, the Schur algebra $S^{X}(n, r)$ and the generalized Schur algebra $S(\pi)$, are isomorphic as algebras. Similar arguments work for $G^{0}$.

By Corollary 3.7 and Proposition 3.9, the two algebras have the same dimension, and so do the two coalgebras. Thus it suffices to show that $K[G]_{r}^{0}$ is contained in $O_{\pi}(K[G])$ as a subcoalgebra. By definition, $O_{\pi}(K[G])$ is the sum of all $K G$-submodules of $K[G]$ belonging to $\pi$. Since $\pi=\pi^{X}(n, r)$ is saturated, it is the set of highest weights of simple composition factors of $E^{\otimes r}$ over $G$. That means $E^{\otimes r}$ belongs to $\pi$ as a $K G$-module. By Lemma 3.10, the coefficient space $K[G]_{r}^{0}$ of $E^{\otimes r}$ also belongs to $\pi$. Hence $K[G]_{r}^{0}$ is contained in $O_{\pi}(K[G])$. Since the coalgebra structure of both coalgebras comes from that of $K[G]$, the inclusion preserves their coalgebra structure.

Remark 3.12. When $r=0, \pi^{X}(n, 0)=\{0\}=\pi_{0}^{X}(n, 0)$ is always saturated, and the Schur algebra $S^{X}(n, 0)=K$ is a generalized Schur algebra in any type.

When $r \geqslant 1$, the weight sets $\pi^{B}(n, r)$ and $\pi_{0}^{B}(n, r)$ of type $B_{m}(m \geqslant 2)$ are not saturated by Lemma 3.8. By comparing the dimension of the Schur algebra $S^{B}(n, r)$ and a generalized Schur algebra, it is clear that $S^{B}(n, r)$ has no chance to be a generalized Schur algebra over $S O_{n}$ or $S O_{n}^{0}$.

In type $A$ the result is actually due to Green [16]. Donkin defined generalized Schur algebras in such a way that Schur algebras of type $A$ provided important examples. Donkin proved the statement for Schur algebras of type $C$ in [9], in a different way. Recently Doty and $\mathrm{Hu}[13]$ provided a different proof of the result in type $D$.

We say an algebra is indecomposable if it cannot be expressed as a direct sum of two proper subalgebras.

Corollary 3.13. In types $A_{m}(m \geqslant 1), C_{m}(m \geqslant 2)$ and $D_{m}(m \geqslant 4)$, any indecomposable generalized Schur algebra $S(\pi)$ of the classical group $G$ is a quotient of $S^{X}(n, r)$ for some 
integer $r \geqslant 0$.

Proof. Let $\pi_{1} \subseteq \pi_{2}$ be two finite saturated subsets of $\Lambda^{+}\left(X_{m}\right)_{\text {int }}$. Then by definition $O_{\pi_{1}}(K[G])$ is a subcoalgebra of $O_{\pi_{2}}(K[G])$. Dually $S\left(\pi_{1}\right)$ is a quotient of $S\left(\pi_{2}\right)$.

Notice that $\Lambda^{+}\left(X_{m}\right)_{\text {int }}=\bigcup_{r \geqslant 0} \pi^{X}(n, r)$. In type $A$, we have $\pi^{X}(n, r) \subseteq \pi^{X}(n, r+n)$ and dominant weights of different degrees are not compatible with respect to the dominance order. In types $C$ and $D$, we have $\pi^{X}(n, r) \subseteq \pi^{X}(n, r+2)$ and dominant weights of even degrees are not compatible with those of odd degrees. Therefore in types $A, C$ and $D$, a finite saturated subset $\pi \subseteq \Lambda^{+}\left(X_{m}\right)_{\text {int }}$ such that $S(\pi)$ is indecomposable must be contained in some $\pi^{X}(n, r)$. So $S(\pi)$ is a quotient of $S\left(\pi^{X}(n, r)\right)$, which is isomorphic to $S^{X}(n, r)$ by Theorem 3.11 .

However the proof is not valid for $G^{0}$. Since there are more dominant weights in $\Lambda_{0}^{+}\left(X_{m}\right)_{\text {int }}$ than $\bigcup_{r \geqslant 0} \pi_{0}^{X}(n, r)$, there exists $\pi \subseteq \Lambda_{0}^{+}\left(X_{m}\right)$ int which cannot be contained in any $\pi^{X}(n, r)$.

\section{Schur algebras and hyperalgebras}

In $\S 4.1$ we construct inverse systems from Schur algebras of a fixed type by using the coalgebra approach. In $\S 4.2$ we prove that the corresponding hyperalgebra is contained in the inverse limit integrally as a subalgebra, see Theorem 4.8. In $\S 4.3$ we discuss the quasi-heredity of Schur algebras.

\subsection{Construction of inverse systems}

Let us fix a classical type $X_{m}$ and the corresponding group of similitudes $G^{0}$. Take a 1dimensional polynomial representation of $G^{0}$ with representation map $\rho: K G^{0} \longrightarrow K$. It is irreducible, and hence homogeneous. Let $r_{0}$ be the degree, and $\mu$ the highest weight. Write $K_{\mu}$ for the representation space. The coefficient function of $K_{\mu}$, denoted by $f_{0}$, is a polynomial in $K\left\langle G^{0}\right\rangle_{r_{0}}$ such that $\rho(g)=f_{0}(g) \in K \cong \operatorname{End}\left(K_{\mu}\right)$ for any group element $g \in G^{0}$. Recall that $K\left\langle G^{0}\right\rangle_{r_{0}}=K\left[c_{i j}\right]_{r_{0}} / I\left\langle G^{0}\right\rangle_{r_{0}}$ is a coalgebra with coalgebra structure inherited from that of $K\left[c_{i j}\right]_{r_{0}}$ (see $\S 2.3$ ), where $I\left\langle G^{0}\right\rangle_{r_{0}}=\left\{c \in K\left[c_{i j}\right]_{r_{0}}: c(g)=0, \forall g \in G^{0}\right\}$.

Lemma 4.1. (1) The comultiplication $\Delta$ of $K\left\langle G^{0}\right\rangle_{r_{0}}$ sends $f_{0}$ to $f_{0} \otimes f_{0}$.

(2) For any integer $r \geqslant 0$, multiplying $f_{0}$ gives rise to a coalgebra injection $\left(\cdot f_{0}\right)$ : $K\left\langle G^{0}\right\rangle_{r} \longrightarrow K\left\langle G^{0}\right\rangle_{r+r_{0}}$.

Proof. (1) By $\S 2.4$, the coefficient space $\operatorname{cf}\left(K_{\mu}\right)$ is a subcoalgebra of $K\left\langle G^{0}\right\rangle_{r_{0}}$. For any elements $g$ and $h$ in $G^{0}, \Delta\left(f_{0}\right)(g \otimes h)=f_{0}(g \cdot h)=\rho(g \cdot h)=\rho(g) \cdot \rho(h)=f_{0}(g) \cdot f_{0}(h)$. That means $\Delta\left(f_{0}\right)=f_{0} \otimes f_{0}$. 
(2) By (1) multiplying $f_{0}$ induces a coalgebra map from $K\left[c_{i j}\right]_{r}$ to $K\left[c_{i j}\right]_{r+r_{0}}$. This map is injective because there is no zero divisor in the polynomial ring. Note that the product of $f_{0}$ and $I\left\langle G^{0}\right\rangle_{r}$ lies inside $I\left\langle G^{0}\right\rangle_{r+r_{0}}$. Hence multiplying $f_{0}$ induces a well-defined coalgebra map $\left(\cdot f_{0}\right): K\left\langle G^{0}\right\rangle_{r} \longrightarrow K\left\langle G^{0}\right\rangle_{r+r_{0}}$. Let $c$ be a polynomial in $K\left[c_{i j}\right]_{r}$ such that the product $c \cdot f_{0}=0$ in $K\left\langle G^{0}\right\rangle_{r+r_{0}}$. Namely $c \cdot f_{0} \in I\left\langle G^{0}\right\rangle_{r+r_{0}}$. Thus $0=\left(c \cdot f_{0}\right)(g)=c(g) \cdot f_{0}(g)$ for any $g \in G^{0}$. But $\rho(g)=f_{0}(g) \neq 0$ since $\rho: K G^{0} \longrightarrow K$ is a representation. Then $c(g)=0$ for any $g \in G^{0}$. That is, the polynomial $c$ belongs to $I\left\langle G^{0}\right\rangle_{r}$. Therefore the map $\left(\cdot f_{0}\right): K\left\langle G^{0}\right\rangle_{r} \longrightarrow K\left\langle G^{0}\right\rangle_{r+r_{0}}$ is injective.

By taking the linear dual one obtains a surjection between Schur algebras $\left(\cdot f_{0}\right)^{*}: S^{X}(n, r+$ $\left.r_{0}\right) \longrightarrow S^{X}(n, r)$ (due to Corollary 3.2). The surjection gives rise to the inverse system of Schur algebras as follows.

Proposition 4.2. (1) For each $r=0,1, \ldots, r_{0}-1,\left\{S^{X}\left(n, r+r_{0} k\right): k \geqslant 0\right\}$ is an inverse system. We write $\varliminf_{k \geqslant 0} S^{X}\left(n, r+r_{0} k\right)$ for the inverse limit.

(2) The surjection $\left(\cdot f_{0}\right)^{*}$ induces a full embedding of the category $M^{X}(n, r) \simeq \bmod \left(S^{X}(n, r)\right)$ into the category $M^{X}\left(n, r+r_{0}\right) \simeq \bmod \left(S^{X}\left(n, r+r_{0}\right)\right)$, which sends $V$ in $M^{X}(n, r)$ to $V \otimes_{K} K_{\mu}$. We denote this functor by $\left(\cdot f_{0}\right)^{*}$ too.

Proof. (1) For $0 \leqslant r \leqslant r_{0}-1$, whenever $0 \leqslant k_{1} \leqslant k_{2}$ there exists a surjection $S^{X}(n, r+$ $\left.r_{0} k_{2}\right) \longrightarrow S^{X}\left(n, r+r_{0} k_{1}\right)$ by a $\left(k_{2}-k_{1}\right)$-fold composition of $\left(\cdot f_{0}\right)^{*}$. Hence $\left\{S^{X}\left(n, r+r_{0} k\right)\right.$ : $k \geqslant 0\}$ is an inverse system indexed by non-negative integers with respect to the natural order.

(2) As an algebra surjection, $\left(\cdot f_{0}\right)^{*}$ naturally induces a full embedding between their module categories (in the opposite direction). Take any $V$ in $M^{X}(n, r)$ with $K$-basis $\left\{v_{i}\right\}$ and coefficient functions $\left\{r_{i j}\right\} \in K\left\langle G^{0}\right\rangle_{r}$, so that $s \cdot v_{j}=\sum_{i} r_{i j}(s) v_{i}$ for any $s \in S^{X}(n, r)$. Then $\left(\cdot f_{0}\right)^{*}(V)$, the image of $V$ in $M^{X}\left(n, r+r_{0}\right)$, has the same $K$-basis $\left\{v_{i}\right\}$ as $V$ and coefficient functions $r_{i j} \cdot f_{0} \in K\left\langle G^{0}\right\rangle_{r+r_{0}}$. By identifying $v_{i}$ of $\left(\cdot f_{0}\right)^{*}(V)$ with $v_{i} \otimes 1$ of $V \otimes_{K} K_{\mu}$, it is clear that $\left(\cdot f_{0}\right)^{*}(V) \cong V \otimes_{K} K_{\mu}$ in $M^{X}\left(n, r+r_{0}\right)$.

We have two examples for the above procedure. Firstly, consider the representation of $G^{0}$ sending a group element to its determinant. This is a $n$-homogeneous polynomial representation with coefficient function det $=\sum_{\sigma \in \sum_{n}} \operatorname{sgn}(\sigma) \prod_{i=1}^{n} c_{i, \sigma(i)}$ and highest weight $\lambda_{0}=\varepsilon_{1}+\varepsilon_{2}+\ldots+\varepsilon_{n}$. We have surjections (.det) ${ }^{*}: S^{X}(n, r+n) \longrightarrow S^{X}(n, r)$ for $r \geqslant 0$. On the dominant weight level, adding $\lambda_{0}$ provides an injection from $\pi_{0}^{X}(n, r)$ to $\pi_{0}^{X}(n, r+n)$. For $0 \leqslant r \leqslant n-1$, the set of Schur algebras $\left\{S^{X}(n, r+n k): k \geqslant 0\right\}$ forms an inverse system.

In types $B, C$ and $D$, consider the representation of $G^{0}$ sending a group element $g$ to $c_{0}(g)$, where $c_{0}$ is the polynomial of degree 2 defined in $\S 2$. This is a 2-homogeneous polynomial 
representation with coefficient function $c_{0}$ and highest weight $\varepsilon_{0}=\varepsilon_{1}+\varepsilon_{n}$. We have surjections $\left(\cdot c_{0}\right)^{*}: S^{X}(n, r+2) \longrightarrow S^{X}(n, r)$ for $r \geqslant 0$. And adding $\varepsilon_{0}$ provides an injection from $\pi_{0}^{X}(n, r)$ to $\pi_{0}^{X}(n, r+2)$. The set $\left\{S^{X}(n, r+2 k): k \geqslant 0\right\}$ forms an inverse system for $r=0$ and $r=1$.

Lemma 4.3. Let $f_{0}$ be the polynomial $\operatorname{det}$ (in types $A, B, C, D$ ), or $\mathrm{c}_{0}$ (in types $B, C, D$ ). For $\lambda \in \pi_{0}^{X}(n, r)$, the functor $\left(\cdot f_{0}\right)^{*}: M^{X}(n, r) \longrightarrow M^{X}\left(n, r+r_{0}\right)$ sends the Weyl module $W(\lambda)$ and the simple module $L(\lambda)$ to $W(\lambda+\mu)$ and $L(\lambda+\mu)$ respectively.

Proof. We prove the case of Weyl modules when $f_{0}=\operatorname{det}, r_{0}=n$ and $\mu=\lambda_{0}$. The other cases follows by similar arguments. By [7], any tensor product of Weyl modules of $G^{0}$ has a filtration with sections being Weyl modules. Now $K_{\lambda_{0}}$ is a simple Weyl module, hence $W(\lambda) \otimes_{K} K_{\lambda_{0}}$ is filtered by Weyl modules. Since $\lambda+\lambda_{0}$ is a highest weight in the tensor product, the Weyl module $W\left(\lambda+\lambda_{0}\right)$ occurs as a quotient of $W(\lambda) \otimes_{K} K_{\lambda_{0}}$. Note that $W(\lambda)$ and $W\left(\lambda+\lambda_{0}\right)$ restrict to the same Weyl module on $G$ (see the argument before Corollary 3.7). In particular $\operatorname{dim} W(\lambda)=\operatorname{dim} W\left(\lambda+\lambda_{0}\right)$. But $\operatorname{dim} W(\lambda)=\operatorname{dim}\left(W(\lambda) \otimes_{K} K_{\lambda_{0}}\right)$. It follows that $W(\lambda) \otimes_{K} K_{\lambda_{0}} \cong W\left(\lambda+\lambda_{0}\right)$.

By Remark 2.3, we have $(\operatorname{det})^{2}=\left(\mathrm{c}_{0}\right)^{n}$ in type $B_{m}$ and hence $\left((\cdot \operatorname{det})^{*}\right)^{2}=\left(\left(\cdot \mathrm{c}_{0}\right)^{*}\right)^{n}$. In type $C_{m}$ and $D_{m}$, we have det $=\left(\mathrm{c}_{0}\right)^{m}$ and hence $(\cdot \operatorname{det})^{*}=\left(\left(\cdot \mathrm{c}_{0}\right)^{*}\right)^{m}$. So the two kinds of inverse systems introduced above are compatible (compare Theorem 6.3 in [22]).

Proposition 4.4. In type $B_{m}(m \geqslant 2)$, for any $i=0,1, \ldots, n-1$ and $j=0,1, \varliminf_{k \geqslant 0} S^{X}(n, i+$ $k n)=\varliminf_{k \geqslant 0} S^{X}(n, j+2 k)$. In type $C_{m}(m \geqslant 2)$ and $D_{m}(m \geqslant 4)$, for any $i=0,1, \ldots, n-1$ and $j=0,1, \varliminf_{k \geqslant 0} S^{X}(n, i+k n)=\varliminf_{k \geqslant 0} S^{X}(n, j+2 k)$ for $i \equiv j(\bmod 2)$.

We set $\operatorname{IL}\left(A_{m}\right)=\bigoplus_{i=0}^{n-1} \varliminf_{\lim _{k} \geqslant 0} S^{A}(n, i+k n), \operatorname{IL}\left(B_{m}\right)=\varliminf_{k \geqslant 0} S^{B}(n, i+k n)$ (for any $i$ ), and $\operatorname{IL}\left(X_{m}\right)=\bigoplus_{r=0}^{1} \lim _{k \geqslant 0} S^{X}(n, i+2 k)$ in types $C_{m}$ and $D_{m}$. Since the injections (-det) and $\left(\cdot c_{0}\right)$ between coalgebras are defined integrally, the inverse systems and inverse limits of Schur algebras are also defined integrally.

\subsection{Recovering Hyperalgebras from Schur algebras}

We refer to [20] for the definition of hyperalgebra, where it is called the algebra of distributions. Take an algebraic group $H$ over $K$. Let $\mathcal{I}$ be the argumentation ideal of the coordinate ring $K[H]$ as a Hopf algebra. Namely $\mathcal{I}$ consists of the rational functions on $H$ which annihilate the unit of $H$. The hyperalgebra hy $(H)$ is by definition the union $\bigcup_{k \geqslant 0}\left(K[H] / \mathcal{I}^{k+1}\right)^{*}$. This is a subalgebra of $K[H]^{*}$. When $K=\mathbb{C}$, the hyperalgebra coincides with the universal enveloping algebra $\mathcal{U}(\mathfrak{h})$ of the Lie algebra $\mathfrak{h}$ of $H$. 
Let $V$ be a rational $K H$-module with representation map $\rho: K H \longrightarrow \operatorname{End}_{K}(V)$. Let $\left\{v_{i}\right\}$ be a basis of $V$, and $\left\{r_{i j}\right\}$ the coefficient functions so that $\rho(h) \cdot v_{j}=\sum_{i} r_{i j}(h) v_{i}$ for $h \in H$. By $\S 2.4$, the right $K[H]$-comodule on $V$ is given by $\rho^{*}: V \longrightarrow V \otimes \operatorname{cf}(V) \hookrightarrow V \otimes K[H]$ and $\rho^{*}\left(v_{j}\right)=\sum_{i} v_{i} \otimes r_{i j}$. By $\S 7.11$ in [20], an element $u$ of hy $(H)$ acts on $V$ by composing

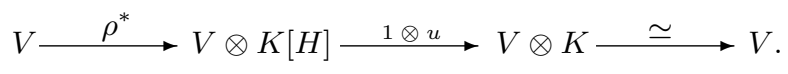

In this way $V$ becomes an hy $(H)$-module. Write $\tilde{\rho}:$ hy $(H) \longrightarrow \operatorname{End}_{K}(V)$ for the representation map. We have that $\tilde{\rho}(u)\left(v_{j}\right)=\sum_{i} u\left(r_{i j}\right) v_{i}$.

Lemma 4.5. The representation map $\rho$ has the same image as the representation map $\tilde{\rho}$.

Proof. The proof is similar to Lemma 2.6. Note that the representation map $\tilde{\rho}$ factors through $\operatorname{cf}(V)^{*}$. That is, there exists an algebra map $\beta: \operatorname{hy}(H) \longrightarrow \operatorname{cf}(V)^{*}$ such that $\tilde{\rho}=\tau \circ \beta$, where $\tau: \operatorname{cf}(V)^{*} \longrightarrow \operatorname{End}_{K}(V)$ is the structure map of $V$ as a faithful $\operatorname{cf}(V)^{*}$-module. In fact, any $u \in \operatorname{hy}(H) \subset K[H]^{*}$ is a linear function on $K[H]$, and hence a linear function on $\operatorname{cf}(V)$. And this defines the map $\beta$. Now it suffices to show the surjectivity of $\beta$. Equivalently, for any $c \in \operatorname{cf}(V)$, if $\beta(u)(c)=0$ for all $u \in \operatorname{hy}(H)$, then $c=0$. If $c$ does not belong to $\mathcal{I}$ and is nonzero, then the counit of the Hopf algebra $K[H]$ acts on $c$ nontrivially. If $c \in \mathcal{I}$ and is nonzero, say $c \in \mathcal{I}^{k} \backslash \mathcal{I}^{k+1}$ for some $k \geqslant 1$, then there exists an element in $\left(K[H] / \mathcal{I}^{k+1}\right)^{*}$ acting on $c$ nontrivially.

When considering the classical group $G$ and the polynomial representation $E^{\otimes r}(r \geqslant 1)$, we have representation maps $\rho^{r}: K G \longrightarrow \operatorname{End}_{K}\left(E^{\otimes r}\right)$ and $\tilde{\rho}^{r}: \operatorname{hy}(G) \longrightarrow \operatorname{End}_{K}\left(E^{\otimes r}\right)$. By Lemma $4.5, \tilde{\rho^{r}}$ factors through the Schur algebra $S^{X}(n, r)=\operatorname{Im}\left(\rho^{r}\right)$.

The next lemma shows that the surjections $(\cdot \operatorname{det})^{*}$ and $\left(\cdot c_{0}\right)^{*}$ between Schur algebra, constructed in the previous subsection, are compatible with $\rho^{r}$ and $\tilde{\rho}^{r}$.

Lemma 4.6. The following diagrams are commutative (where (·det)* is defined for all types and $\left(\cdot \cdot_{0}\right)^{*}$ defined for types $\left.B, C, D\right)$ :
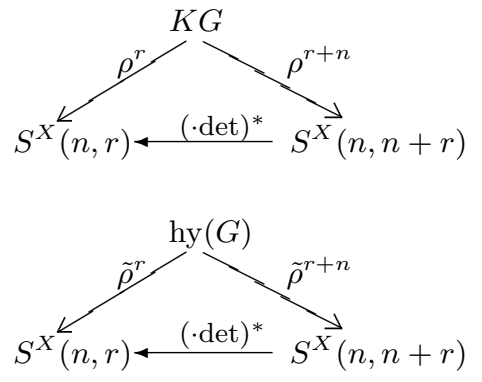
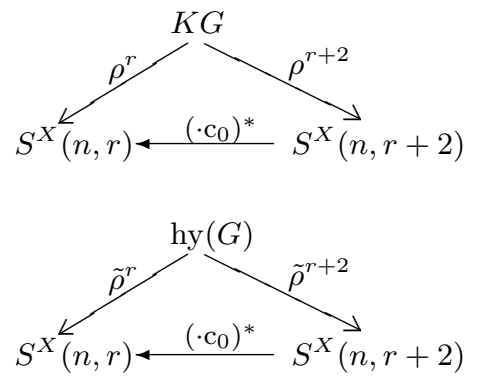
Proof. For the first diagram, one has to show that $\rho^{r}(g)$ and $(\cdot \operatorname{det})^{*} \circ \rho^{r+n}(g)$ are equal in $S^{X}(n, r)$ for any $g \in G$. That is, they have the same evaluation on the coalgebra $K[G]_{r}^{0}$. By definition (.det)* is the dual of (·det) $: K[G]_{r}^{0}=K\left\langle G^{0}\right\rangle_{r} \longrightarrow K\left\langle G^{0}\right\rangle_{r+n}=K[G]_{r+n}^{0}$. For any $c \in K[G]_{r}^{0}$, we have that $\left((\cdot \operatorname{det})^{*} \circ \rho^{r+n}(g)\right)(c)=\left(\rho^{r+n}(g)\right)(c \cdot \operatorname{det})=(c \cdot \operatorname{det})(g)=c(g) \cdot \operatorname{det}(g)=$ $c(g)=\left(\rho^{r}(g)\right)(c)$. The other diagrams follow similarly by using the equalities det $=1$ (in all types) and $\mathrm{c}_{0}=1$ (in types $B, C$ and $D$ ) on $G$ and $\operatorname{hy}(G)$.

Remark 4.7. The Schur algebra can also be defined as the image of the hyperalgebra hy $\left(G^{0}\right)$ of $G^{0}$. However, the diagrams in Lemma 4.6 are not commutative for $K G^{0}$ or hy $\left(G^{0}\right)$. Because both equalities $\operatorname{det}=1$ and $\mathrm{c}_{0}=1$ fail on $G^{0}$ and hy $\left(G^{0}\right)$.

By the universal property of inverse limit, there exists an algebra homomorphism from the hyperalgebra hy $(G)$ to the inverse limit $\mathrm{IL}\left(X_{m}\right)$ of Schur algebras. Write $\tau$ for this map.

Theorem 4.8. The hyperalgebra is contained in the inverse limit of Schur algebras, i.e. $\tau$ : $\operatorname{hy}_{K}(G) \longrightarrow \operatorname{IL}\left(X_{m}\right)$ is an injection.

Proof. First we will show that the representation $\oplus_{r \geqslant 0} E^{\otimes r}$ over hy $(G)$ is faithful. The coefficient space of $\oplus_{r \geqslant 0} E^{\otimes r}$ over $G$ is $K[G]$. By definition the hyperalgebra hy $(G)$ is contained in the dual of $K[G]$. So for any nonzero element $u$ in hy $(G)$, there exist some $c$ in $K[G]$ such that $c(u) \neq 0$. This means that the kernel of the representation map hy $(G) \longrightarrow \operatorname{End}_{K}\left(\oplus_{r} \geqslant 0 E^{\otimes r}\right)$ is trivial. Namely $\oplus_{r \geqslant 0} E^{\otimes r}$ is faithful over hy $(G)$.

Notice that hy $(G)$ sends $E^{\otimes r}$ to $E^{\otimes r}$. Therefore for any nonzero element $u$ in hy $(G)$, there exists a non-negative integer $N$ such that $u$ acts nontrivially on $E^{\otimes N}$. Namely the representation map $\tilde{\rho}^{N}: \operatorname{hy}(G) \longrightarrow \operatorname{End}_{K}\left(E^{\otimes N}\right)$ sends $u$ to a nonzero element in $S^{X}(n, N)$. Note that in type $B$, the integer $N$ can always be chosen to be even. This implies that $\tau$ sends $u$ to a nonzero element in $\operatorname{IL}\left(X_{m}\right)$, i.e. $\tau: \operatorname{hy}(G) \longrightarrow \operatorname{IL}\left(X_{m}\right)$ is injective.

In the quantum case, this embedding was developed by Beilinson, Lusztig and MacPherson [1] in type $A$ and was conjectured by Oehms [23] in type $C$. Recently Doty [12] studied the inverse system of generalized Schur algebras of linear algebraic groups and recovered the corresponding quantized enveloping algebra.

\subsection{Quasi-heredity of Schur algebras}

(Integral) quasi-hereditary algebras were introduced in [2, 3], see also [21]. Let $S$ be a finite dimensional quasi-hereditary algebra over $K$, and $\pi$ the index set of isomorphism classes of simple $S$-modules. Suppose $\pi=\left\{\lambda_{1}, \lambda_{2}, \ldots, \lambda_{m}\right\}$ with the partial order ' $>$ ' satisfying that 
$\lambda_{i}>\lambda_{j}$ implies $i<j$. For $1 \leqslant i \leqslant m$, write $e_{\lambda_{i}}$ for a primitive idempotent of $S$ corresponding to $\lambda_{i}$. Then

$$
0 \subset S e_{\lambda_{1}} S \subset S\left(e_{\lambda_{1}}+e_{\lambda_{2}}\right) S \subset \ldots \subset S\left(e_{\lambda_{1}}+e_{\lambda_{2}}+\ldots+e_{\lambda_{m}}\right) S=S
$$

provides a socalled heredity chain of $S$. Each section $S\left(e_{\lambda_{1}}+\ldots+e_{\lambda_{i}}\right) S / S\left(e_{\lambda_{1}}+\ldots+e_{\lambda_{i-1}}\right) S$ is actually a direct sum of copies of the standard module $\Delta\left(\lambda_{i}\right)$. The heredity chain shows that $S$ has a filtration of standard modules, and that $\operatorname{Ext}^{1}\left(\Delta\left(\lambda_{i}\right), \Delta\left(\lambda_{j}\right)\right) \neq 0$ implies $i>j$. Moreover, the algebra $S$ is called integral quasi-hereditary if it is integrally defined and if the heredity chain is integrally defined as well.

Recall that Theorem 3.11 shows Schur algebras of types $A, C$ and $D$ are generalized Schur algebras. Since generalized Schur algebras are integral quasi-hereditary by $[8,10]$, we have the following.

Corollary 4.9. In types $A_{m}(m \geqslant 1), C_{m}(m \geqslant 2)$ and $D_{m}(m \geqslant 4)$, for any integer $r \geqslant 0$, Schur algebras $S^{X}(n, r)$ is integral quasi-hereditary with index set $\pi^{X}(n, r)$ with respect to the dominance order.

The standard module $\Delta(\lambda)$ of $S^{X}(n, r)$, for $\lambda \in \pi^{X}(n, r)$ (and $\pi_{0}^{X}(n, r)$ ), is the Weyl module $W(\lambda)$ for $G$ (and $G^{0}$ respectively).

Proposition 4.10. In types $A_{m}(m \geqslant 1), C(m \geqslant 2)$ and $D_{m}(m \geqslant 4)$, the surjections (-det)* and $\left(\cdot c_{0}\right)^{*}$ between Schur algebras are compatible with the integral quasi-hereditary structure. That is, the kernels of $(\cdot \operatorname{det})^{*}$ and $\left(\cdot \mathrm{c}_{0}\right)^{*}$ are ideals in their heredity chains.

Proof. We only prove the statement for the surjection (-det)* $: S^{X}(n, r+n) \longrightarrow S^{X}(n, r)$. The proof for $\left(\cdot c_{0}\right)^{*}$ is similar. As a rational $K G$-module, also as its own regular module, $S^{X}(n, r)$ has composition factors belonging to $\pi^{X}(n, r)$. By the first commutative diagram in Lemma 4.6, $S^{X}(n, r)$ is a quotient $K G$-module of $S^{X}(n, r+n)$ belonging to $\pi^{X}(n, r)$.

We view the module category $\bmod \left(S^{X}(n, r)\right)$ as a full subcategory of the category of rational representations of $G$. By Proposition $4.2(2)$, (.det)* induces a full embedding of $\bmod \left(S^{X}(n, r)\right)$ into $\bmod \left(S^{X}(n, r+n)\right)$. The one-dimensional representation $K_{\text {det }}$ is the trivial representation over $G$. Hence by Lemma 4.3 , (-det)* sends the standard module $\Delta(\lambda)$ of $S^{X}(n, r)$, for $\lambda \in \pi^{X}(n, r)$, to the standard module $\Delta(\lambda)$ of $S^{X}(n, r+n)$. Moreover, it sends the regular module $S^{X}(n, r) \in \bmod \left(S^{X}(n, r)\right)$ to $S^{X}(n, r) \in \bmod \left(S^{X}(n, r+n)\right)$, which has a filtration of the standard modules $\Delta(\lambda)$ for $\lambda \in \pi^{X}(n, r)$.

Note that the index set $\pi^{X}(n, r)$ is a saturated subset of $\pi^{X}(n, r+n)$. For $S:=S^{X}(n, r+n)$ for short, write $e$ for the sum of the $e_{\lambda}$ 's with $\lambda$ running through $\pi^{X}(n, r+n) \backslash \pi^{X}(n, r)$. By 
the hereditary structure of $S$, the ideal $S e S$ generated by $e$ lies in the kernel of $(\cdot \operatorname{det})^{*}$ : $S \longrightarrow S^{X}(n, r)$. Note that when $K=\mathbb{C}$, the Schur algebras are semisimple (Corollary $4.3[22])$, and each section of the hereditary chain is just $\Delta(\lambda)^{\oplus d_{\lambda}}$, where $d_{\lambda}=\operatorname{dim}_{\mathbb{C}} \Delta(\lambda)$. So $\operatorname{dim}_{\mathbb{C}}(S / S e S)=\sum_{\lambda \in \pi^{X}(n, r)} d_{\lambda}^{2}$. Since Schur algebras are integral quasi-hereditary, $\operatorname{dim}_{K}(S / S e S)$ is independent of the field $K$, and it equals $\operatorname{dim}_{K}\left(S^{X}(n, r)\right)$ by Corollary 3.7. Therefore $\operatorname{Ker}\left((\cdot \operatorname{det})^{*}\right)=S e S$, which is an ideal in the heredity chain of $S$.

For dominant weights $\lambda$ and $\mu$ of $G^{0}$, let $[\lambda: \mu]$ be the multiplicity of the simple module $L(\mu)$ in the Weyl module $W(\lambda)$ of $G^{0}$. We have the following analogue of James' column removal Theorem [19], and an even stronger version in types $C, D$. Recall that $\lambda_{0}=\varepsilon_{1}+\varepsilon_{2}+$ $\ldots+\varepsilon_{n}$, and $\varepsilon_{0}=\varepsilon_{1}+\varepsilon_{n}$.

Corollary 4.11. Let $\lambda$ and $\mu$ be dominant weights in $\pi_{0}^{X}(n, r)$. In types $A, C$ and $D$, we have that $[\lambda: \mu]=\left[\lambda+\lambda_{0}: \mu+\lambda_{0}\right]$. Moreover in types $C$ and $D$, we have that $[\lambda: \mu]=\left[\lambda+\varepsilon_{0}: \mu+\varepsilon_{0}\right]$.

Proof. The multiplicity of $L(\mu)$ in $W(\lambda)$ over $G^{0}$ equals the multiplicity of $L(\mu)$ in $\Delta(\lambda)$ over $S^{X}(n, r)$. By Corollary 4.10, the full embedding of categories $(\cdot \operatorname{det})^{*}: M^{X}(n, r) \longrightarrow$ $M^{X}(n, r+n)$ and $\left(\cdot c_{0}\right)^{*}: M^{X}(n, r) \longrightarrow M^{X}(n, r+2)$ preserve the multiplicity.

We would like to mention that this is a special case of Corollary 2 in [6, page 232], where Donkin considers all reductive algebraic groups.

\section{$5 \quad$ Examples of Schur algebras}

In $\S 5.1$ we calculate the Schur algebras of $\mathrm{SO}_{2}$ and $\mathrm{O}_{2}$, and compare the Schur algebras of $S P_{2}$ and $S_{3}$ with $S L_{2}$. In $\S 5.2$ we show the Schur-Weyl duality in types $B, C$ and $D$ when the parameter $r=2$, and obtain as byproducts the algebra structure of $S^{X}(n, 2)$ and some information on decomposition numbers. The symplectic Schur-Weyl duality for arbitrary parameter has been proved by Dipper, Doty and $\mathrm{Hu}[5]$, while the orthogonal version is proved now by Doty and $\mathrm{Hu}[13]$.

\subsection{Calculation and comparison of some Schur algebras}

Recall that the base field $K$ is algebraically closed of characteristic not 2 . These examples generalize those in $[22]$ where $K=\mathbb{C}$.

Example. The classical group of type $C_{1}$ is the symplectic group $S P_{2}=\left\{M \in \mathcal{M}_{2}(K)\right.$ : $\left.M^{t r} J^{\prime} M=J^{\prime}\right\}$, where $J^{\prime}=\left(\begin{array}{cc}0 & 1 \\ -1 & 0\end{array}\right)$. Notice that $M^{t r} J^{\prime} M=J^{\prime}$ if and only if $\operatorname{det}(M)=1$. Hence $S P_{2}=S L_{2}$ the special linear group, and $S^{C}(2, r)=S^{A}(2, r)$ for any $r \geqslant 0$. 
Example. The classical group of type $D_{1}$ is the special orthogonal group

$$
S O_{2}=\left\{M \in \mathcal{M}_{2}(K): M^{t r} J M=J=M J M^{t r}, \operatorname{det}(M)=1\right\},
$$

where $J=\left(\begin{array}{ll}0 & 1 \\ 1 & 0\end{array}\right)$. The coordinate ring $K\left[S O_{2}\right]=K\left[c_{i j}\right] / I\left(S O_{2}\right)$, where $I\left(S_{2}\right)$ is generated by $\left\{c_{11} c_{22}-c_{12} c_{21}-1, c_{11} c_{22}+c_{12} c_{21}-1,2 c_{11} c_{12}, 2 c_{21} c_{22}, 2 c_{11} c_{21}, 2 c_{12} c_{22}\right\}$. It follows that $I\left(S O_{2}\right)^{0}=\left\langle c_{12}, c_{21}\right\rangle$ and $K\left[S O_{2}\right]^{0}=K\left[c_{i j}\right] /\left\langle c_{12}, c_{21}\right\rangle \cong K\left[c_{11}, c_{22}\right]$. For $r \geqslant 0$, the homogeneous part $K\left[S O_{2}\right]_{r}^{0}$ has a basis $\left\{c_{11}^{i} c_{22}^{r-i}: i=0,1, \ldots, r\right\}$. Hence $S^{D}(2, r)$, isomorphic to $\left(K\left[S O_{2}\right]_{r}^{0}\right)^{*}$, has an integral dual basis $\left\{\xi_{1^{i} 2^{r-i}, 1^{i} 2^{r-i}}=\left(c_{11}^{i} c_{22}^{r-i}\right)^{*}: i=0,1, \ldots, r\right\}$ (Green's notation in [16]). It is a subalgebra of $S^{A}(2, r)$ isomorphic to $K^{\oplus(r+1)}$.

Example. The classical group of type $B_{1}$ is the algebraic group $S_{3}$. We will show that the Schur algebra $S^{B}(3, r)$ is isomorphic to $S^{A}(2,2 r)$ when $r \geqslant 2$. By [22], the two algebras are isomorphic over $\mathbb{C}$ and hence have the same dimension. So it suffices to construct a surjective coalgebra map between their linear dual $K\left\langle S O_{3}^{0}\right\rangle_{r}$ and $K\left\langle G L_{2}\right\rangle_{2 r}$.

By definition

$$
S O_{3}^{0}=\left\{M \in \mathcal{M}_{3}(K): M^{t r} J M=M J M^{t r}=s J, \operatorname{det}(M)=t, t, s \in K^{*}\right\},
$$

where $J=\left(\begin{array}{lll}0 & 0 & 1 \\ 0 & 1 & 0 \\ 1 & 0 & 0\end{array}\right)$. The coalgebra $K\left\langle S O_{3}^{0}\right\rangle=K\left[c_{i j}\right] / I\left\langle S O_{3}^{0}\right\rangle$, and the ideal $I\left\langle S O_{3}^{0}\right\rangle$ is generated by quadratic relations $\left\{c_{1}(i, j), c_{2}(i, j): \forall j \neq i^{\prime}\right\} \cup\left\{c_{1}\left(i, i^{\prime}\right)-c_{1}(1,3), c_{2}\left(j, j^{\prime}\right)-\right.$ $\left.c_{1}(1,3): \forall i, j\right\}$, where $i^{\prime}=4-i$. For example $c_{1}(1,1)=2 c_{31} c_{11}+c_{21}^{2}, c_{1}(1,2)=c_{31} c_{12}+$ $c_{21} c_{22}+c_{11} c_{32}, c_{1}(2,2)-c_{1}(1,3)=c_{12} c_{32}+c_{22} c_{22}+c_{32} c_{12}-c_{11} c_{33}-c_{21} c_{23}-c_{31} c_{13}$. Tо distinguish from $S O_{3}^{0}$, we write $\left\{d_{i j}\right\}_{i, j=1,2}$ for the coordinate functions of $G L_{2}$. Recall that $K\left\langle G L_{2}\right\rangle=K\left[d_{i j}\right]($ see $\S 2.3)$.

Sending entries to the corresponding entries:

$$
\left(\begin{array}{ccc}
c_{11} & c_{12} & c_{13} \\
c_{21} & c_{22} & c_{23} \\
c_{31} & c_{32} & c_{33}
\end{array}\right)-\longrightarrow\left(\begin{array}{ccc}
d_{11}^{2} & -\sqrt{2} d_{11} d_{12} & -d_{12}^{2} \\
-\sqrt{2} d_{11} d_{21} & d_{11} d_{22}+d_{12} d_{21} & \sqrt{2} d_{12} d_{22} \\
-d_{21}^{2} & \sqrt{2} d_{21} d_{22} & d_{22}^{2}
\end{array}\right)
$$

we obtain a linear map from $K\left[c_{i j}\right]_{1}$ to $K\left[d_{i j}\right]_{2}$, denoted by $\phi$. It is actually a coalgebra map, i.e. $\Delta\left(\phi\left(c_{i j}\right)\right)=\phi\left(\Delta\left(c_{i j}\right)\right)$, for any $i, j=1,2,3$. For instance when $i=j=1$,

$$
\begin{aligned}
\Delta\left(\phi\left(c_{11}\right)\right) & =\Delta\left(d_{11}^{2}\right)=\left(\Delta\left(d_{11}\right)\right)^{2}=\left(d_{11} \otimes d_{11}+d_{12} \otimes d_{21}\right)^{2} \\
& =d_{11}^{2} \otimes d_{11}^{2}+2 d_{11} d_{12} \otimes d_{11} d_{21}+d_{12}^{2} \otimes d_{21}^{2} \\
\phi\left(\Delta\left(c_{11}\right)\right) & =\phi\left(c_{11} \otimes c_{11}+c_{12} \otimes c_{21}+c_{13} \otimes c_{31}\right) \\
& =\phi\left(c_{11}\right) \otimes \phi\left(c_{11}\right)+\phi\left(c_{12}\right) \otimes \phi\left(c_{21}\right)+\phi\left(c_{13}\right) \otimes \phi\left(c_{31}\right) \\
& =d_{11}^{2} \otimes d_{11}^{2}+\left(-\sqrt{2} d_{11} c_{12}\right) \otimes\left(-\sqrt{2} d_{11} d_{21}\right)+d_{12}^{2} \otimes d_{21}^{2} \\
& =d_{11}^{2} \otimes d_{11}^{2}+2 d_{11} d_{12} \otimes d_{11} d_{21}+d_{12}^{2} \otimes d_{21}^{2} .
\end{aligned}
$$


Extending $\phi$ by $\phi^{2}\left(c_{1} c_{2}\right)=\phi\left(c_{1}\right) \phi\left(c_{2}\right)$, for any $c_{1}, c_{2} \in K\left[c_{i j}\right]_{1}$, we obtain a coalgebra map $\phi^{2}: K\left[c_{i j}\right]_{2} \longrightarrow K\left[d_{i j}\right]_{4}$. We claim that $\phi^{2}$ is surjective.

Any monomial $d$ in $K\left[d_{i j}\right]_{4}$ is a product $d_{1} d_{2}$ with $d_{1}$ and $d_{2}$ having degree 2 . If $d_{1}=d_{11} d_{22}$ and $d_{2}=d_{11}^{2}$, then $d_{1} d_{2}=\phi^{2}\left(c_{11} c_{22}-\frac{1}{2} c_{12} c_{21}\right)$ lies in $\operatorname{Im}\left(\phi^{2}\right)$. If $d_{1}=d_{11} d_{22}$ and $d_{2}=d_{22}^{2}$, then $d_{1} d_{2}=\phi^{2}\left(c_{22} c_{33}-\frac{1}{2} c_{23} c_{32}\right)$ lies in $\operatorname{Im}\left(\phi^{2}\right)$. If $d_{1}=d_{11} d_{22}$ and $d_{2} \neq d_{11}^{2}$ or $d_{22}^{2}$, write $d_{2}=d_{k_{1} l_{1}} d_{k_{2} l_{2}}$ (where $k_{1}, k_{2}, l_{1}, l_{2} \in\{1,2\}$ ). Then either both $d_{11} d_{k_{1} l_{1}}$ and $d_{22} d_{k_{2} l_{2}}$ or both $d_{11} d_{k_{2} l_{2}}$ and $d_{22} d_{k_{1} l_{1}}$ lie in $\operatorname{Im}(\phi)$, and hence the product $d_{1} d_{2}$ lies in $\operatorname{Im}\left(\phi^{2}\right)$. Similarly if $d_{1}=d_{12} d_{21}$, then $d_{1} d_{12}^{2}=\phi^{2}\left(-c_{22} c_{13}+\frac{1}{2} c_{12} c_{23}\right)$ and $d_{1} d_{21}^{2}=\phi^{2}\left(-c_{22} c_{31}+\frac{1}{2} c_{21} c_{32}\right)$ lie in $\operatorname{Im}\left(\phi^{2}\right)$. If $d_{1}=d_{12} d_{21}$ and $d_{2} \neq d_{12}^{2}$ or $d_{21}^{2}$, then by rearranging the order in the product, it is not hard to see that $d_{1} d_{2}$ lies in $\operatorname{Im}\left(\phi^{2}\right)$. Finally if neither $d_{1}$ nor $d_{2}$ belongs to $\left\{d_{11} d_{22}, d_{12} d_{21}\right\}$, then the product naturally lies in $\operatorname{Im}\left(\phi^{2}\right)$. In this way we have shown that $\phi^{2}$ is surjective.

Extending $\phi$ inductively we have surjective coalgebra maps $\phi^{r}: K\left[c_{i j}\right]_{r} \longrightarrow K\left[d_{i j}\right]_{2 r}$ for $r \geqslant 2$. Now it is straightforward to check all quadratic relations in $I\left\langle S O_{3}^{0}\right\rangle$ belong to the kernel of $\phi^{2}$. For instance,

$$
\begin{aligned}
\phi^{2}\left(2 c_{31} c_{11}+c_{21}^{2}\right) & =-2 d_{21}^{2} d_{11}^{2}+\left(-\sqrt{2} d_{11} d_{21}\right)^{2}=0, \\
\phi^{2}\left(c_{31} c_{12}+c_{21} c_{22}+c_{11} c_{32}\right) & =\left(-d_{21}^{2}\right)\left(-\sqrt{2} d_{11} d_{12}\right)+\left(-\sqrt{2} d_{11} d_{21}\right)\left(d_{11} d_{22}+d_{12} d_{21}\right)+d_{11}^{2}\left(\sqrt{2} d_{21} d_{22}\right) \\
& =0 .
\end{aligned}
$$

Since $\phi^{r}$ is defined to preserve product, $I\left\langle S O_{3}^{0}\right\rangle_{r}$ lies in the kernel of $\phi^{r}(r \geqslant 2)$. So we have obtained a surjective coalgebra map $K\left\langle S O_{3}^{0}\right\rangle_{r} \longrightarrow K\left\langle G L_{2}\right\rangle_{2 r}$ for any $r \geqslant 2$.

Let us define Schur algebras for the orthogonal group $O_{n}=\left\{M \in \mathcal{M}_{n}(K): M^{t r} J M=\right.$ $\left.J=M J M^{t r}\right\}$, where $J$ is any symmetric invertible matrix and $n \geqslant 2$. The special orthogonal group $S O_{n}$ is the subgroup of $O_{n}$ with determinant 1. The group of orthogonal similitudes is $O_{n}^{0}=\left\{M \in \mathcal{M}_{n}(K): M^{t r} J M=s J=M J M^{t r}, 0 \neq s \in K\right\}$. As subgroups of $G L_{n}$, the algebraic groups $O_{n}$ and $O_{n}^{0}$ act on $E$ and on the tensor space $E^{\otimes r}(r \geqslant 1)$. The corresponding Schur algebra, denoted by $S^{o}(n, r)$, is defined to be the image of the representation map $K O_{n} \longrightarrow \operatorname{End}_{K}\left(E^{\otimes r}\right)$, which equals the image of $K O_{n}^{0} \longrightarrow \operatorname{End}_{K}\left(E^{\otimes r}\right)$. By convention $S^{o}(n, 0)=K$. Write $K\left[O_{n}\right]_{r}^{0}$ (and $\left.K\left\langle O_{n}^{0}\right\rangle_{r}\right)$ for the coefficient space of $E^{\otimes r}$ over $O_{n}$ (and $O_{n}^{0}$ respectively). It is in fact the restriction of $K\left[c_{i j}\right]_{r}$ to $O_{n}$ (and $O_{n}^{0}$ respectively). By Lemma 2.6, the orthogonal Schur algebra $S^{o}(n, r)$ is isomorphic to the dual of the coalgebras $K\left[O_{n}\right]_{r}^{0}$ and $K\left\langle O_{n}^{0}\right\rangle_{r}$. Take $J$ as in Remark 2.1 .

Proposition 5.1. When $n$ is odd, we have $S^{o}(n, r)=S^{B}(n, r)$ for $r \geqslant 0$. When $n$ is even, we have $S^{o}(n, r)=S^{D}(n, r)$ for $r<n / 2$. 
Proof. Write $I\left(O_{n}^{0}\right)$ for the ideal of $K\left[c_{i j}\right]$ generated by $\left\{c_{1}\left(i, i^{\prime}\right)-c_{2}\left(j, j^{\prime}\right): \forall i, j\right\}$ and $\left\{c_{1}(i, j), c_{2}(i, j): \forall j \neq i^{\prime}\right\}$. This is a homogeneous ideal. The coalgebra $K\left\langle O_{n}^{0}\right\rangle_{r}$ is the quotient of $K\left[c_{i j}\right]_{r}$ factoring out the homogeneous part $I\left(O_{n}^{0}\right)_{r}=I\left(O_{n}^{0}\right) \cap K\left[c_{i j}\right]_{r}$. It is clear that when $n$ is odd, $I\left(O_{n}^{0}\right)_{r}=I\left(S O_{n}^{0}\right)_{r}$ for all non-negative integer $r$, and when $n$ is even, $I\left(O_{n}^{0}\right)_{r}=I\left(S O_{n}^{0}\right)_{r}$ for $r<n / 2$. The statement follows.

Example. When $n=2, I\left(O_{2}^{0}\right)=\left\langle c_{11}, c_{22}\right\rangle \cdot\left\langle c_{12}, c_{21}\right\rangle$, and $K\left[O_{2}^{0}\right] \cong K\left[c_{i j}\right] /\left\langle c_{11}, c_{22}\right\rangle \oplus$ $K\left[c_{i j}\right] /\left\langle c_{12}, c_{21}\right\rangle \cong K\left[c_{12}, c_{21}\right] \oplus K\left[c_{11}, c_{22}\right]$. For $r \geqslant 1, K\left[O_{2}^{0}\right]_{r}$ has a basis $\left\{c_{12}^{i} c_{21}^{r-i}, c_{11}^{i} c_{22}^{r-i}\right.$ : $i=0,1, \ldots, r\}$. Hence $S^{o}(2, r)$ has an integral dual basis $\left\{\xi_{1^{i} 2^{r-i}, 2^{i} 1^{r-i}}, \xi_{1^{i} 2^{r-i}, 1^{i} 2^{r-i}}: i=\right.$ $0,1, \ldots, r\}$. This is a subalgebra of $S^{A}(2, r)$ of dimension $2(r+1)$ and

$$
S^{o}(2, r) \cong \begin{cases}\left(\mathcal{M}_{2}(K)\right)^{\oplus \frac{r+1}{2}} & \text { if } \mathrm{r} \text { is odd } \\ \left(\mathcal{M}_{2}(K)\right)^{\oplus \frac{r}{2}} \oplus K^{\oplus 2} & \text { if } \mathrm{r} \text { is even. }\end{cases}
$$

\subsection{Schur-Weyl duality}

We take $J$ to be the identity matrix to define the orthogonal group, and $J^{\prime}$ the same as in Remark 2.1 to define the symplectic groups. Let $\left\{v_{i}: i=1,2, \ldots, n\right\}$ be the natural basis of $E$. Then $\left\{v_{i} \otimes v_{j}: i, j=1,2, \ldots, n\right\}$ is a basis of $E \otimes E$. We write $v_{i j}=v_{i} \otimes v_{j}$ for short.

The Brauer algebra $B_{2}(\delta), \delta \in K \backslash\{0\}$, is a three-dimensional commutative $K$-algebra with basis $\left\{\Delta_{0}\right.$ (unit), $\left.\Delta_{1}, \Delta_{2}\right\}$ and multiplications

$$
\Delta_{1}^{2}=\Delta_{0}, \quad \Delta_{2}^{2}=\delta \Delta_{2}, \quad \Delta_{1} \Delta_{2}=\Delta_{2} \Delta_{1}=\Delta_{2}
$$

In type $B_{m}$ and $D_{m}$, the parameter $\delta=n$. The Brauer algebra $B_{2}(n)$ acts on $E \otimes E$ on the right with $\Delta_{0}$ acting as identity, $\Delta_{1}$ sending $v_{i j}$ to $v_{j i}$, and $\Delta_{2}$ sending $v_{i j}$ to $\delta_{i j} \sum_{k=1}^{n} v_{k k}$. In type $C_{m}, \delta=-n$. The Brauer algebra $B_{2}(-n)$ acts on $E \otimes E$ on the right with $\Delta_{0}$ acting as identity, $\Delta_{1}$ sending $v_{i j}$ to $-v_{j i}$, and $\Delta_{2}$ sending $v_{i j}$ to $\epsilon(i) \delta_{j i^{\prime}} \sum_{k=1}^{n} \epsilon\left(k^{\prime}\right) v_{k k^{\prime}}$, where $i^{\prime}=n+1-i$.

The main result in this subsection is the following.

Theorem 5.2. Let $G$ be a classical group of type $B_{m}(m \geqslant 2), C_{m}(m \geqslant 2)$ or $D_{m}(m \geqslant 4)$. Then the actions on $E \otimes E$ by $G$ on the left and by $B_{2}(\delta)$ on the right commute with each other, where $\delta=n$ in type $B, D$, and $-n$ in type $C$. Moreover the double centralizer property (i.e. the Schur-Weyl duality) holds:

$$
\begin{aligned}
S^{X}(n, 2) & =\operatorname{End}_{B_{2}(\delta)}(E \otimes E), \\
B_{2}(\delta) & =\operatorname{End}_{K G}(E \otimes E)=\operatorname{End}_{S^{X}(n, 2)}(E \otimes E) .
\end{aligned}
$$


By Proposition 5.1, in type $B$ and $D$, Theorem 5.2 remains true if the special orthogonal group is replaced by the corresponding orthogonal group. Let us prove the result in this case by a series of Lemmas $5.3-5.8$. Write $p(\neq 2)$ for the characteristic of $K$.

Lemma 5.3. (Theorem 1.7.7 [14]) Let $B$ be a K-algebra, and $1=e_{1}+e_{2}+\ldots+e_{l}$ be a primitive central decomposition of the identity. Namely $e_{i}$ 's are primitive central and pairwise orthogonal idempotents. Then $B$ has a block decomposition $B=B_{1} \oplus B_{2} \oplus \ldots \oplus B_{l}$ where $B_{i}=B e_{i}=e_{i} B$ for each $1 \leqslant i \leqslant l$. That is, every right $B$-module $V$ is isomorphic to $V_{1} \oplus V_{2} \oplus \ldots \oplus V_{l}$, where $V_{i}=V e_{i}$ and $B_{i}$ acts on $V_{j}$ non-trivially if and only if $i=j$. Moreover $\operatorname{End}_{B}(V) \cong \bigoplus_{i=1}^{l} \operatorname{End}_{B_{i}}\left(V_{i}\right)$.

Lemma 5.4. (1) Suppose $p$ does not divide $n$, and write $e_{1}=\frac{1}{2} \Delta_{0}+\frac{1}{2} \Delta_{1}-\frac{1}{n} \Delta_{2}, e_{2}=\frac{1}{n} \Delta_{2}$ and $e_{3}=\frac{1}{2} \Delta_{0}-\frac{1}{2} \Delta_{1}$. Then $\Delta_{0}=e_{1}+e_{2}+e_{3}$ is a primitive central decomposition, and the Brauer algebra $B_{2}(n)$ is isomorphic to $K \oplus K \oplus K$. We write $L(1), L(2)$ and $L(3)$ for the simple projective right $B_{2}(n)$-modules corresponding to $e_{1}, e_{2}$ and $e_{3}$ respectively.

(2) Suppose $p$ divides $n$, and write $\widetilde{e_{1}}=\frac{1}{2} \Delta_{0}+\frac{1}{2} \Delta_{1}$ and $\widetilde{e_{2}}=\frac{1}{2} \Delta_{0}-\frac{1}{2} \Delta_{1}$. Then $\Delta_{0}=$ $\widetilde{e_{1}}+\widetilde{e_{2}}$ is a primitive central decomposition, and $B_{2}(n)$ is isomorphic to $K[X] /\left(X^{2}\right) \oplus K$. We write $\widetilde{L}(1)$ and $\widetilde{L}(2)$ for the simple modules corresponding to $\widetilde{e_{1}}$ and $\widetilde{e_{2}}$ respectively, and $\widetilde{P}(1)=B_{2}(n) \widetilde{e_{1}}$ the projective cover of $\widetilde{L}(1)$.

Note that $B_{2}(n) \widetilde{e_{1}}$ has $K$-basis $\left\{\frac{1}{2} \Delta_{0}+\frac{1}{2} \Delta_{1}, \Delta_{2}\right\}$ and is isomorphic to $K[X] /\left(X^{2}\right)$ by identifying $\Delta_{2}$ with $X$.

Lemma 5.5. (1) When $p$ does not divide $n$, as a right $B_{2}(n)$-module $E \otimes E$ is isomorphic to $L(1)^{\oplus\left(\frac{n^{2}+n}{2}-1\right)} \oplus L(2) \oplus L(3)^{\oplus \frac{n^{2}-n}{2}}$.

(2) When $p$ divides $n$, as a right $B_{2}(n)$-module $E \otimes E$ is isomorphic to $\widetilde{L}(1)^{\oplus\left(\frac{n^{2}+n}{2}-2\right)} \oplus$ $\widetilde{P}(1) \oplus \widetilde{L}(2)^{\oplus \frac{n^{2}-n}{2}}$.

Proof. (1) By direct calculation, $(E \otimes E) e_{1}$ has $K$-dimension $\frac{n^{2}+n}{2}-1$ with basis $\left\{v_{i i}-\right.$ $\left.\frac{1}{n} \sum_{k=1}^{n} v_{k k^{\prime}}: 1 \leqslant i \leqslant n\right\} \cup\left\{\frac{1}{2}\left(v_{i j}+v_{j i}\right): 1 \leqslant j<i \leqslant n\right\},(E \otimes E) e_{2}$ is 1-dimensional with basis $\left\{\frac{1}{n} \sum_{k=1}^{n} v_{k k^{\prime}}\right\}$, and $(E \otimes E) e_{3}$ has $K$-dimension $\frac{n^{2}-n}{2}$ with basis $\left\{\frac{1}{2}\left(v_{i j}-v_{j i}\right): 1 \leqslant j<i \leqslant n\right\}$. The result follows from Lemma 5.3 and Lemma 5.4(1).

(2) By direct calculation, $(E \otimes E) \widetilde{e_{1}}$ has $K$-dimension $\frac{n^{2}+n}{2}$ with basis $\left\{\frac{1}{2}\left(v_{i j}+v_{j i}\right)\right.$ : $1 \leqslant j \leqslant i \leqslant n\}$. It has $\frac{n^{2}+n}{2}-2$ direct summands isomorphic to $\widetilde{L}(1)$, with basis $\left\{\frac{1}{2}\left(v_{i j}+\right.\right.$ $\left.\left.v_{j i}\right): 1 \leqslant i<j \leqslant n\right\} \cup\left\{v_{i i}-v_{n n}: 1 \leqslant i \leqslant n-2\right\}$. Their complement is isomorphic to $\widetilde{P}(1)$ with basis $\left\{v_{n n}, \sum_{k=1}^{n} v_{k k^{\prime}}\right\}$. Similarly $(E \otimes E) \widetilde{e_{2}}$ has dimension $\frac{n^{2}-n}{2}$ with basis $\left\{\frac{1}{2}\left(v_{i j}-v_{j i}\right): 1 \leqslant j<i \leqslant n\right\}$. The result follows from Lemma 5.3 and Lemma 5.4(2). 
Lemma 5.6. (1) When $p$ does not divide $n$, the algebra $\operatorname{End}_{B_{2}(n)}(E \otimes E)$ is semisimple and isomorphic to $\mathcal{M}_{\frac{n^{2}+n}{2}-1}(K) \oplus K \oplus \mathcal{M}_{\frac{n^{2}-n}{2}}(K)$.

(2) When $p$ divides $n$, the algebra $\operatorname{End}_{B_{2}(n)}(E \otimes E)$ is quasi-hereditary with quiver

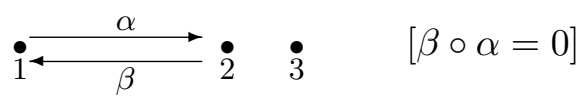

and the partial order $1>2$. The corresponding simple modules $S(1), S(2)$ and $S(3)$ have dimensions $\frac{n^{2}+n}{2}-2,1$ and $\frac{n^{2}-n}{2}$ respectively.

(3) In any case the dimension of the endomorphism algebra is

$$
\left(\frac{n^{2}+n}{2}-1\right)^{2}+1+\left(\frac{n^{2}-n}{2}\right)^{2}=\left(\begin{array}{c}
n^{2}+1 \\
2
\end{array}\right)-(n+2)(n-1) .
$$

Proof. Follows from Lemma 5.5.

Lemma 5.7. The actions on $E \otimes E$, by $O_{n}$ on the left and by $B_{2}(n)$ on the right, commute.

Proof. It suffices to check that $\left(g v_{i j}\right) \Delta_{2}=g\left(v_{i j} \Delta_{2}\right)$, for any $g$ in $O_{n}$ and any $i, j$. Recall that $g=\left(a_{i j}\right) \in O_{n}$ if and only if $g^{t r} g=I=g g^{t r}$. That is, $\sum_{k=1}^{n} a_{i k} a_{j k}=\delta_{i j}=\sum_{k=1}^{n} a_{k i} a_{k j}$. Therefore

$$
\begin{aligned}
\left(g v_{i j}\right) \Delta_{2} & =\left(g \cdot\left(v_{i} \otimes v_{j}\right)\right) \Delta_{2}=\left(g v_{i} \otimes g v_{j}\right) \Delta_{2}=\left(\sum_{k, l=1}^{n} a_{k i} a_{l j} v_{k l}\right) \Delta_{2} \\
& =\sum_{k, l=1}^{n} a_{k i} a_{l j}\left(v_{k l} \Delta_{2}\right)=\sum_{k=1}^{n} a_{k i} a_{k j}\left(v_{k k} \Delta_{2}\right) \\
& =\sum_{k=1}^{n} a_{k i} a_{k j}\left(\sum_{l=1}^{n} v_{l l}\right)=\delta_{i j} \sum_{l=1}^{n} v_{l l}, \\
g\left(v_{i j} \Delta_{2}\right) & =g\left(\delta_{i j} \sum_{k=1}^{n} v_{k k}\right)=\delta_{i j} \sum_{k=1}^{n} g v_{k} \otimes g v_{k}=\delta_{i j} \sum_{k=1}^{n} \sum_{p, q=1}^{n} a_{p k} a_{q k} v_{p q} \\
& =\delta_{i j} \sum_{p, q=1}^{n}\left(\sum_{k=1}^{n} a_{p k} a_{q k}\right) v_{p q}=\delta_{i j} \sum_{p=1}^{n} v_{p p}=\left(g v_{i j}\right) \Delta_{2} .
\end{aligned}
$$

Lemma 5.8. In types $B_{m}$ and $D_{m}$, we have the following:

$$
\begin{aligned}
(1) & S^{X}(n, 2)=\operatorname{End}_{B_{2}(n)}(E \otimes E) \\
(2) & B_{2}(n)=\operatorname{End}_{S^{X}(n, 2)}(E \otimes E) .
\end{aligned}
$$

Proof. (1) By Lemma 5.7, the Schur algebra $S^{X}(n, 2)$, which is the image of the representation map $K O_{n} \longrightarrow \operatorname{End}_{K}(E \otimes E)$, becomes a subalgebra of the endomorphism algebra. They must coincide because they have the same dimension by Lemma 5.6 and Corollary 3.7. 
(2) When $p$ does not divide $n$, the endomorphism algebra is isomorphic to $K \oplus K \oplus K$ by (1) and Lemma 5.6(1). When $p$ divides $n, E \otimes E$ has the module structure

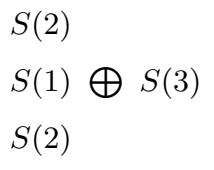

over $S^{X}(n, 2)$. So the endomorphism algebra is isomorphic to $K[X] /\left(X^{2}\right) \oplus K$. In both cases, the endomorphism algebra is isomorphic to $B_{2}(n)$ by Lemma 5.4. But $B_{2}(n)$ is a subalgebra of $\operatorname{End}_{S^{X}(n, 2)}(E \otimes E)=\operatorname{End}_{O_{n}}(E \otimes E)$ by Lemma 5.7. So they are equal.

The proof for symplectic type is similar. Let us list the process by Lemma $5.9-5.12$ without giving proofs.

Lemma 5.9. Suppose $p$ does not divide $n$.

(1) The Brauer algebra $B_{2}(-n)$ is semisimple and isomorphic to $K \oplus K \oplus K$ with three primitive central idempotents: $e_{1}=\frac{1}{2} \Delta_{0}-\frac{1}{2} \Delta_{1}, e_{2}=-\frac{1}{n} \Delta_{2}$ and $e_{3}=\frac{1}{2} \Delta_{0}+\frac{1}{2} \Delta_{1}+\frac{1}{n} \Delta_{2}$.

(2) Write $L(1), L(2)$ and $L(3)$ for the simple projective right $B_{2}(-n)$-modules corresponding to $e_{1}, e_{2}$ and $e_{3}$ respectively. As a right $B_{2}(-n)$-module $E \otimes E$ is isomorphic to $L(1)^{\oplus\left(\frac{n^{2}+n}{2}\right)} \oplus L(2) \oplus L(3)^{\oplus \frac{n^{2}-n}{2}-1}$.

(3) The endomorphism algebra $\operatorname{End}_{B_{2}(-n)}(E \otimes E)$ is semisimple and isomorphic to $\mathcal{M}_{\frac{n^{2}+n}{2}}(K) \oplus$ $K \oplus \mathcal{M}_{\frac{n^{2}-n}{2}-1}(K)$.

Lemma 5.10. Suppose $p$ divides $n$.

(1) The Brauer algebra $B_{2}(-n)$ is isomorphic to $K[X] /\left(X^{2}\right) \oplus K$ with two primitive central idempotents: $\widetilde{e_{1}}=\frac{1}{2} \Delta_{0}-\frac{1}{2} \Delta_{1}$ and $\widetilde{e_{2}}=\frac{1}{2} \Delta_{0}+\frac{1}{2} \Delta_{1}$.

(2) Write $\widetilde{L}(1)$ and $\widetilde{L}(2)$ for the simple right $B_{2}(-n)$-modules corresponding to $\widetilde{e_{1}}$ and $\widetilde{e_{2}}$ respectively, and $\widetilde{P}(2)$ for $B_{2}(-n) \widetilde{e_{2}}$ the projective cover of $\widetilde{L}(2)$. Note that $\widetilde{L}(1)$ is both simple and projective. As a right $B_{2}(-n)$-module, $E \otimes E$ is isomorphic to $\widetilde{L}(1)^{\oplus \frac{n^{2}+n}{2}} \oplus \widetilde{P}(2) \oplus$ $\widetilde{L}(2)^{\frac{n^{2}-n}{2}-2}$.

(3) The endomorphism algebra $\operatorname{End}_{B_{2}(-n)}(E \otimes E)$ is quasi-hereditary with quiver

$$
i \quad \stackrel{\alpha}{\stackrel{\beta}{\longleftarrow}} ; \quad[\alpha \circ \beta=0]
$$

and the partial order $3>2$. The corresponding simple modules $S(1), S(2)$ and $S(3)$ have dimensions $\frac{n^{2}+n}{2}, 1$ and $\frac{n^{2}-n}{2}-2$ respectively.

Lemma 5.11. The dimension of the endomorphism algebra $\operatorname{End}_{B_{2}(-n)}(E \otimes E)$ is $\left(\frac{n^{2}+n}{2}\right)^{2}+$ $1+\left(\frac{n^{2}-n}{2}-1\right)^{2}=\left(\begin{array}{c}n^{2}+1 \\ 2\end{array}\right)-(n-2)(n+1)$. 
Lemma 5.12. (1) The actions on $E \otimes E$, by $S P_{n}$ on the left and by $B_{2}(-n)$ on the right, are commutative.

(2) As algebras over $K, S^{C}(n, 2)=\operatorname{End}_{B_{2}(-n)}(E \otimes E)$ and $\operatorname{End}_{S^{C}(n, 2)}(E \otimes E)=B_{2}(-n)$.

In types $B, C$ and $D$, the weight set $\pi^{X}(n, 2)=\left\{2 \varepsilon_{1}, \varepsilon_{1}+\varepsilon_{2}, 0\right\}$ by Proposition 3.5, and $2 \varepsilon_{1} \unrhd \varepsilon_{1}+\varepsilon_{2} \unrhd 0$ under the dominance order. By the dimension formula of Weyl modules given in Lemma 5.1 [22], the vertices 1,2 and 3 in the quiver of $S^{X}(n, 2)$ correspond to the dominant weights $2 \varepsilon_{1}, 0$ and $\varepsilon_{1}+\varepsilon_{2}$ respectively. Since modules of the Schur algebra $S^{X}(n, 2)$ is naturally viewed as polynomial representations of the corresponding classical group, we have the following.

Corollary 5.13. Suppose $p \neq 2$. For groups $S O_{n}$ and $O_{n}(n=5$ or $n \geqslant 7)$, the decomposition number $\left[2 \varepsilon_{1}: \varepsilon_{1}+\varepsilon_{2}\right]=0,\left[\varepsilon_{1}+\varepsilon_{2}: 0\right]=0$. And $\left[2 \varepsilon_{1}: 0\right]=0$ when $p$ does not divide $n$, and 1 when $p$ divides $n$. For groups $S P_{n}(n \geqslant 4$, even $)$, we have $\left[2 \varepsilon_{1}: \varepsilon_{1}+\varepsilon_{2}\right]=0,\left[2 \varepsilon_{1}: 0\right]=0$. And $\left[\varepsilon_{1}+\varepsilon_{2}: 0\right]=0$ when $p$ is does not divide $n$, and 1 when $p$ divides $n$.

Equivalently, for (special) orthogonal similitudes, we have $\left[2 \varepsilon_{1}: \varepsilon_{1}+\varepsilon_{2}\right]=0$, $\left[\varepsilon_{1}+\varepsilon_{2}\right.$ : $\left.\varepsilon_{0}\right]=0$. And $\left[2 \varepsilon_{1}: \varepsilon_{0}\right]=0$ when $p$ does no divide $n$, and 1 when $p$ divides $n$. For symplectic similitudes, we have $\left[2 \varepsilon_{1}: \varepsilon_{1}+\varepsilon_{2}\right]=0,\left[2 \varepsilon_{1}: \varepsilon_{0}\right]=0$. And $\left[\varepsilon_{1}+\varepsilon_{2}: \varepsilon_{0}\right]=0$ when $p$ does not divide $n$, and 1 when $p$ divides $n$.

Combining Corollary 5.13 with Corollary 4.11, we hope it provides some interesting information on decomposition numbers of classical groups.

\section{References}

[1] A.A. Beilinson, G. Lusztig, R. MacPherson, A geometric setting for the quantum deformation of $G L_{n}$, Duke Math. J. 61 (1990) 655-677.

[2] E. Cline, B. Parshall, L.L. Scott, Finite dimensional algebras and highest weight categories, J. reine angew. Math. 391 (1988) 85-99.

[3] E. Cline, B. Parshall, L.L. Scott, Integral and graded quasi-hereditary algebras I, J. Algebra 131 (1990) 126-160.

[4] R. Dipper, S. Doty, The rational Schur algebra, preprint, 2005, arXiv:math.RT/0511663.

[5] R. Dipper, S. Doty, J. Hu, Brauer algebras, symplectic Schur algebras and Schur-Weyl duality, preprint, arXiv:math.RT/0503545. 
[6] S. Donkin, A note on decomposition numbers for reductive groups, J. Algebra 80 (1983) $226-234$.

[7] S. Donkin, Rational representations of algebraic groups: Tensor products and filtrations, Lecture notes in Mathematics 1140, Springrt-Verlag, Berlin, 1985.

[8] S. Donkin, Schur algebras and related algebras I, J. Algebra 104 (1986) 310-328.

[9] S. Donkin, Good filtrations of rational modules for reductive groups, Proceedings of symposia in pure mathematics, Volume 47 (1987) 69-80.

[10] S. Donkin, Schur algebras and related algebras III: integral representations, Math. Proc. Camb. Phil. Soc. 116 (1994) 37-55.

[11] S. Doty, Polynomial representations, algebraic monoids, and Schur algebras of classical type, Journal of Pure and Applied Algebras 123 (1998) 165-199.

[12] S. Doty, Constructing quantized enveloping algebras via inverse limits of finite dimensional algebras, preprint, arXiv:math.QA/0711.2764.

[13] S. Doty, J. Hu, Schur-Weyl duality for orthogonal groups, preprint, arXiv:math.RT/0712.1944.

[14] Y. Drozd, V.V. Dirichenko, Finite dimensional dimensiona algebras, With an Appendix by V. Dlab, Translated from the Russian by V. Dlab, Springer-Verlag, 1994.

[15] J.A. Green, Locally finite representations, J. Algebra 41 (1976) 137-171.

[16] J.A. Green, Polynomial representataions of $G L_{n}$, Lecture Notes in Mathematics 830, Berlin/Heidelberg/New York, Springer-Verlag, 1980.

[17] T. Hayashi, Quantum deformation of classical groups, Publ. Res. Inst. Math. Sci. 28 (1992) 57-81.

[18] J.E. Humphreys, Introduction to Lie algebras and representation theory, Springer, Graduate texts in mathematics 9, Springer-Verlag, 1972.

[19] G.D. James, On the decomposition matrices of the symmestric groups III, J. Algebra 71 (1981) 115-122.

[20] J.C. Jantzen, Representations of algebraic groups, Boston: academic press, 1987.

[21] M. Klucznik, S. Koenig, Characteristic tilting modules over quasi-hereditary algebras, Course note (from the compact course given in March, 1998), 1-64, available from http://www.mi.uni-koeln.de/ skoenig/.

[22] Q. Liu, Schur algebras of classical groups, J. Algebra 301 (2006) 867-887. 
[23] S. Oehms, Symplectic q-Schur algebras, PhD theis (English version), University of Stuttgart, Shaker Verlag Aachen, 1997. 